IFT-UAM/CSIC-13-01

\title{
Holographic Type II Goldstone Bosons
}

\author{
Irene Amado ${ }^{a, 1}$, Daniel Areán ${ }^{b, e}, 2$, Amadeo Jimenez-Albac $\left\{^{3}\right.$,

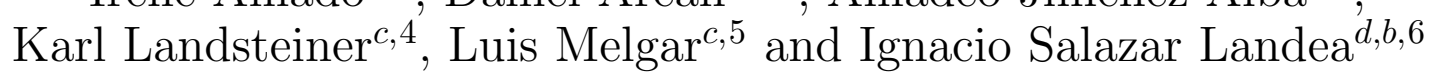 \\ ${ }^{a}$ Department of Physics, Technion, Haifa 32000, Israel \\ ${ }^{b}$ International Centre for Theoretical Physics (ICTP), Strada Costiera 11; I 34014 Trieste, Italy \\ ${ }^{c}$ Instituto de Física Teórica IFT-UAM/CSIC, Universidad Autónoma de Madrid, 28049 \\ Cantoblanco, Spain \\ ${ }^{d}$ Instituto de Física La Plata (IFLP) and Departamento de Física Universidad Nacional de La \\ Plata, CC 67, 1900 La Plata, Argentina \\ ${ }^{e}$ INFN - Sezione di Trieste, Strada Costiera 11; I 34014 Trieste, Italy
}

\begin{abstract}
The Goldstone theorem implies the appearance of an ungapped mode whenever a continuous global symmetry is spontaneously broken. In general it does not say anything about the precise form of the dispersion relation nor does it imply that there is one massless mode for each broken symmetry generator. It is a well-established fact that even for relativistic field theories in the presence of a chemical potential Goldstone modes with quadratic dispersion relation, the type II Goldstone bosons, appear in the spectrum. We develop two holographic models that feature type II Goldstone modes as part of the quasinormal mode spectrum. The models are based on simple generalizations with $U(2)$ symmetry of the well-studied holographic s-wave superfluid. Our results include Goldstone modes without broken generators but with unusual realization of symmetries and a frequency dependent conductivity of striking resemblance to the one of Graphene.
\end{abstract}

\footnotetext{
${ }^{1}$ irene.r.amado@gmail.com

2 arean@sissa.it

3 amadeo.j@gmail.com

${ }^{4}$ karl.landsteiner@csic.es

${ }^{5}$ luis.melgar@csic.es

${ }^{6}$ peznacho@gmail.com
} 


\section{Introduction}

The AdS/CFT correspondence has proved to be a powerful tool to study strongly coupled quantum systems, with applications to QCD and condensed matter systems. Among the applications to condensed matter physics one of the most important examples is the construction of the so called holographic superfluids [1, 2, 3].

The ingredients of such a model are a charged black hole describing a CFT at finite temperature and charge density. At sufficiently low temperature (or high chemical potential) a charged scalar field develops an expectation value and triggers a symmetry breaking phase transition towards a superfluid phase. In the simplest model the order parameter is a scalar and therefore we speak of an s-wave superfluid. Furthermore it is possible to go to a decoupling limit in which the fluctuations of the bulk metric are suppressed. The dynamics in this limit is completely specified by the fluctuations of the gauge field and the scalar field [3]. Generalizations to p-wave [4] and d-wave [5, 6] superconductors, for which the order parameter has angular momentum $l=1$ and $l=2$ respectively are also known. Much of this development is reviewed in [7, 8].

The strength of the gauge/gravity correspondence is that it allows to study the real time dynamics of strongly coupled field theories rather easily. Linear response theory captures the behavior of a quantum system after an initial, small perturbation. It also applies to the late time behavior when an initially large perturbation has already sufficiently died out and enters the linear regime. The basic ingredient of linear response theory is the retarded Green's function. In the context of the AdS/CFT correspondence it was shown in [9, 10] how to calculate retarded Green's functions by imposing infalling boundary conditions at the black hole horizon. For black holes with non-degenerate horizons the retarded Green's functions are analytic in the upper half of the complexified frequency plane and have (an infinite series of) isolated poles in the lower half. These poles are the holographic quasinormal modes (QNM) of the black hole [11, 12, 13, 14]. Within the QNM spectrum, the ungapped modes play a special role, since they give the dominant contribution to the retarded Green's functions at low frequency and small momentum. Therefore they determine the hydrodynamic description of the system. In this paper we will study the quasinormal mode spectrum of a multi-component generalization of the simple, holographic, non-backreacted s-wave superfluid with particular focus on the low lying and hydrodynamic modes.

The study of the QNMs for the s-wave $U(1)$ superfluid was first carried out in [15]. Since the basic physics of superfluids is the one of spontaneous symmetry breaking it can be expected that known results such as the existence of a Goldstone boson ${ }^{1}$ carry over to the holographic models. Indeed, one of the main results of [15] was that the QNM spectrum in the superfluid phase contains such an ungapped Goldstone mode with dispersion relation $\omega= \pm v_{s} k+O\left(k^{2}\right)$. This mode can also be understood as the sound mode of the superfluid and $v_{s}$ is the sound velocity ${ }^{2}$. In the non-backreacted model these are the

\footnotetext{
${ }^{1}$ It is also often called Nambu-Goldstone boson. For simplicity we will refer to it as Goldstone boson or Goldstone mode throughout the text.

${ }^{2}$ In [16] it was pointed out that this mode corresponds to the fourth sound. Whereas second sound is defined through temperature oscillations fourth sound is the phenomenon of sound propagation in the
} 
only hydrodynamic modes in the broken phase. In the unbroken phase in contrast there exists a single hydrodynamic mode signaling the usual diffusive behavior of a normal fluid. Its dispersion relation is $\omega=-i D k^{2}$, where $D$ is the diffusion constant. The question what happens to this diffusive mode in the broken phase was also answered in [15]: it develops a purely imaginary gap $\omega=-i \gamma-i \tilde{D} k^{2}$. This is quite natural because the single purely imaginary mode can not move off the imaginary axis? 3 . The hydrodynamics of the broken phase is fully captured by the Goldstone mode and the diffusion mode does the simplest thing it can to drop out of the hydrodynamic regime by growing the gap $\gamma$. Since this purely imaginary gapped mode has its origin in the universal diffusive mode of the unbroken phase we expect that it is a universal feature of a large class of superfluids, not only holographic ones. This mode will necessarily dominate the late time response in the order parameter to homogeneous perturbations and in regimes close but below the critical temperature where the gap $\gamma$ is rather small. Therefore the order parameter is bound to show a purely exponential decay towards its equilibrium value without any oscillation. In contrast for lower temperatures where $\gamma$ becomes large there are other low lying QNMs with real and imaginary parts in their frequency. In this low temperature regime the response in the order parameter is then an exponentially damped oscillation rather than a purely imaginary decay. This universal aspect of the late time response of superfluids was also emphasized in recent numerical studies of quenches of holographic superfluids in [17].

In this paper we generalize the results on the QNM spectrum to models with $U(2)$ symmetry. In the first model we simply add a second scalar field of the same mass, we will call this the ungauged model. A second model also includes gauge fields for the whole $U(2)$ symmetry. The difference between the two models is as follows. In the ungauged model only the $U(1)$ symmetry is local in the bulk. It has however a global $S U(2)$ symmetry under which the scalar fields transform as a doublet. According to the holographic dictionary this model contains only one conserved current, corresponding to the single gauge field in the bulk. The dual field theory inherits of course the global $S U(2)$ symmetry of the bulk but this symmetry is not generated by operators in the dual conformal field theory. This is similar to the decoupling limit in which we are working and in which the fluctuations of the metric are suppressed. The dual field theory has then strictly speaking no energy momentum tensor. In usual four dimensional Lagrangian field theories Noether's theorem guarantees that we can always construct a conserved charge generating a given symmetry of the Lagrangian. In holographically defined field theories the existence of a four dimensional Lagrangian is a priori not guaranteed and therefore Noether's theorem does not straightforwardly apply. This is the case here. Although the dual field theory has the $S U(2)$ symmetry (and Poincaré covariance) it does not contain operators generating these symmetries. We can speak of these symmetries

superfluid component only [40. This is the mode that survives the probe limit in which propagation in the normal component of the dual fluid is prohibited. In this model there is no other sound mode so we will simplfy refer to it as the sound mode. Due to the nature of fourth sound it interpolates between second sound at $T=T_{c}$ and normal sound at $T=0$.

${ }^{3}$ Quasinormal modes are bound to come either in pairs $\omega_{n}$ and $\tilde{\omega}_{n}=-\omega_{n}^{*}$ or are fixed on the imaginary axis. This follows from rather generic symmetry considerations for retarded Green's functions, see appendix A. 
as an outer automorphism of the operator algebra of the dual field theory ${ }^{4}$. Physically the difference between the two models is that the ungauged one is a one-component fluid (there is only one notion of charge) whereas the gauged one is a two component fluid. In the latter case the charges are the expectation values of the zero-component of the currents in the Cartan subalgebra of the $U(2)$ symmetry.

Although this ungauged model does not contain conserved currents for the $S U(2)$ symmetry and therefore many of the standard proofs about existence of Goldstone bosons do not strictly apply we find a new ungapped mode in the QNM spectrum of the scalars. This mode is however not a standard Goldstone boson with linear dispersion relation but a so-called type II Goldstone mode whose energy depends quadratically on momentum.

The second model we consider has a scalar field doublet coupled to the full set of $U(2)$ gauge fields. We switch on a chemical potential only for the overall $U(1)$ symmetry. Therefore the high temperature phase has the full $U(2)$ symmetry. At low temperatures this symmetry is broken to $U(1)$. In this model the dual field theory contains currents for all the $U(2)$ symmetries. We can therefore also study the conductivities.

In the context of condensed matter physics it has been pointed out long ago in [18] that such multicomponent superfluids have unusual Goldstone modes with quadratic dispersion relation. In the high energy context such models have been considered as models for Kaon condensation in the color-flavor locked phase of QCD in [19, 20] again emphasizing the existence of the quadratic Goldstone mode. Our gauged holographic model is a straightforward holographic analogue of the model in [19, 20] and indeed we also find the presence of a Goldstone mode with quadratic dispersion relation. Let us also note that in the holographic context a type II Goldstone boson was found before in magnetized D3/D5 defect theory 21].

It seems useful to collect now some of the known theorems on Goldstone bosons (a very useful review on symmetry breaking and Goldstone modes is [22]). First we have of course the actual Goldstone theorem. Its proof assumes the existence of a conserved current $j^{\mu}$ such that the broken charge is $Q=\int d^{d} x j^{0}$ (with $d$ spatial dimensions). The theorem then states that spontaneous breaking of a continuous global symmetry implies the existence of a mode whose energy fulfills

$$
\lim _{k \rightarrow 0} \omega(k)=0
$$

The theorem by itself does not make any statement about the number of these modes, nor does it fix the $k$-dependence of the frequency. In the presence of Poincaré symmetry one can make however a stronger statement, namely that the dispersion relation of the Goldstone mode has to be linear and that the number of Goldstone bosons equals the number of broken generators.

Lorentz symmetry might be absent however, either in principle such as in non-relativistic field theories or the system under consideration might be in a Lorentz symmetry breaking state, such as being at finite temperature or density. In these cases another theorem classifies

\footnotetext{
${ }^{4}$ A string theory example for such a situation is the theory based on the small $\mathcal{N}=4$ superconformal algebra on the world sheet. This algebra possesses a large $S O(4)=S U(2) \times S U(2)$ symmetry acting on the four supercharges of which only one $S U(2)$ is represented through chiral currents on the worldsheet.
} 
Goldstone bosons as type I if their energy vanishes as an odd power of the momentum or as type II if their energy vanishes as an even power of the momentum in the zero momentum limit. The number of type I and type II Goldstone bosons has to fulfill then

$$
n_{I}+2 n_{I I} \geq N_{B G}
$$

where $N_{B G}$ is the number of broken generators [23]. The number of type I and type II Goldstone bosons can be further constrained. Upon assuming that the broken symmetry generators obey $\left\langle\left[Q_{a}, Q_{b}\right]\right\rangle=B_{a b}$ the number of Goldstone bosons has to fulfill [24, 25, 27] (see also [22, 28, 26] for more on counting rules of Goldstone bosons).

$$
n_{I}+n_{I I}=N_{B G}-\frac{1}{2} \operatorname{rank}(B) .
$$

We note that the gauged holographic model fulfills all these theorems. We have in total four symmetry generators. The symmetry is broken from $U(2)$ to $U(1)$ and so there are three broken generators. In the broken phase the charges corresponding to the overall $U(1)$ and the Cartan $U(1)$ generator inside $S U(2)$ receive vacuum expectation values. Therefore the rank of the matrix $B$ is two and so the number of type I and type II Goldstone bosons should add up to two. This is precisely what we find in the QNM spectrum, one ungapped mode with linear dispersion relation and one ungapped mode with quadratic dispersion relation.

We also note that the ungauged model satisfies Goldstone's theorem and the counting rule of Chadha and Nielsen (2). It violates however the more refined counting rule (3). In a strict sense this model only has one symmetry generator since it has only one $U(1)$ gauge field in the bulk. Therefore the counting rule (3) would suggest the existence of only one Goldstone boson, the number of broken generators is one and the matrix $B$ vanishes trivially.

This paper is organized as follows. In section 2 we briefly review a simple field theoretical model featuring type II Goldstone modes. This model has been introduced in the context of Kaon condensation in color-flavor locked QCD. It serves us as inspiration for constructing the holographic models.

Section 3 is devoted to the analysis of the ungauged model. Since the well-known s-wave superconductor is a subsector of both the ungauged and the gauged model we also briefly review first the findings of [15]. Then we show that even with this drastic simplification, i.e. not gauging the global $S U(2)$ symmetry in the bulk, the model presents Goldstone modes with quadratic dispersion relation. Hence, within this model a type II NG boson is found as a consequence of having broken just one charge generator (the one associated to the $U(1)$ symmetry).

In section 4 we study the fully gauged $U(2)$ model. Then we analyze the fluctuation equations to linear order. They decompose into three decoupled sectors. One being the already encountered $U(1)$ s-wave superfluid, the other describing the non-Abelian sector in which the type II Goldstone mode resides and a third one with the unbroken $U(1)$ symmetry. We proceed to study the conductivities which now arrange naturally into a two by two matrix. We show that the diagonal conductivities have delta-functions at zero frequency and are in this sense superconducting. Furthermore upon a change of basis we find a frequency 
dependence that is strikingly similar to the one of Graphene [29]. Furthermore we find indications that for temperatures below $T=0.4 T_{c}$ another instability arises in the gauge field sector leading to an additional p-wave condensate. Then we study the low lying quasinormal modes and analyze the results. We find the type II Goldstone mode and also study the fate of the diffusion modes in the broken phase. Since now two symmetries participate there are two diffusion modes that in the broken phase pair up and can move away from the imaginary axis. We find that this is precisely what happens. Therefore the response in this sector does not show the purely exponential decay induced by the gapped pseudo diffusion mode of the $U(1)$ sector.

We conclude in section 5 with a discussion of our results and an outlook to further possible studies of holographic type II Goldstone modes.

Finally, in appendix A we present some general properties of matrix-valued Green's functions from which constraints on the quasinormal mode spectrum follow . In appendix B we collect technical details on how to actually compute the QNMs for coupled systems.

\section{A field theoretical model with type II Goldstone bo- son}

Motivated by the physics of Kaon condensation in the color-flavor locked phase of QCD the authors of [19, 20] studied QCD at a nonzero chemical potential for strangeness. It was shown that at a critical value of the chemical potential equal to the Kaon mass, Kaon condensation occurs through a continuous phase transition. Moreover, a Goldstone boson with the non-relativistic dispersion relation $\omega \sim p^{2}$ appears in the Kaon condensed phase. To illustrate this fact, they considered the following (Euclidean) toy model:

$$
\mathcal{L}=\left(\partial_{0}+\mu\right) \phi^{\dagger}\left(\partial_{0}-\mu\right) \phi+\partial_{i} \phi^{\dagger} \partial_{i} \phi+M^{2} \phi^{\dagger} \phi+\lambda\left(\phi^{\dagger} \phi\right)^{2}
$$

where $\phi$ is a complex scalar doublet,

$$
\phi=\left(\begin{array}{c}
\phi_{1} \\
\phi_{2}
\end{array}\right)
$$

As long as $\mu<M$ the masses of the four excitations in the model are the positive roots in $\omega$ of

$$
(\omega \pm \mu)^{2}=M^{2}
$$

All are doubly degenerate. It is straightforward to check that at $\mu=M$ the global $U(2)$ symmetry gets broken and the new vacuum can be chosen to be:

$$
\phi=\frac{1}{\sqrt{2}}\left(\begin{array}{l}
0 \\
v
\end{array}\right), \quad \text { with } \quad v^{2}=\frac{\mu^{2}-M^{2}}{\lambda} .
$$


Studying the fluctuations of the doublet $\phi$ around this background one finds two massless and two massive modes with the following dispersion relations:

$$
\begin{aligned}
\omega_{1}^{2} & =\frac{\mu^{2}-M^{2}}{3 \mu^{2}-M^{2}} p^{2}+O\left(p^{4}\right), \\
\omega_{2}^{2} & =6 \mu^{2}-2 M^{2}+O\left(p^{2}\right) \\
\omega_{3}^{2} & =p^{2}-2 \mu \omega_{3} \\
\omega_{4}^{2} & =p^{2}+2 \mu \omega_{4} .
\end{aligned}
$$

If we concentrate on the positive roots we see that $\omega_{1}$ is a normal, linear Goldstone mode. The positive root of equation 10 is

$$
\omega_{3}=\frac{p^{2}}{2 \mu}+O\left(p^{4}\right)
$$

This is the type II Goldstone mode. It has formally a non-relativistic dispersion relation. Since the underlying theory has however Lorentz invariance there is of course also a negative energy mode with quadratic dispersion. This arises as the negative root of $\omega_{4}$. Finally $\omega_{2}$ and $\omega_{4}$ are gapped modes with

$$
\omega_{4}=2 \mu+O\left(p^{2}\right) .
$$

Since the symmetry breaking pattern is $U(2) \rightarrow U(1)$ there are three broken generators but only two massless Goldstone modes in the spectrum. This model fulfills all the counting theorems noted in the introduction. In particular the Chadha-Nielsen rule (2) is exactly saturated. The role of $\omega_{4}$ is special. It is the mode that pairs up with the type II Goldstone mode in the dispersion relations (10) and (11). It has been argued that this mode is a universal feature and that its energy at zero momentum is exact and protected against quantum corrections [28, 30, 31]. The spectrum obtained from this model is summed up in Figure 1. In our holographic models we will look for this special gapped partner mode of the type II Goldstone mode. It will turn out that the gauged and ungauged models differ significantly here: only the mode in the gauged model shows the characteristic linear dependence on the chemical potential.

This simple Lagrangian model serves as our motivation and guideline to construct a holographic model featuring type II Goldstone modes. In fact we can use the same kind of matter Lagrangian in a holographic setup. According to the usual holographic dictionary a local bulk symmetry corresponds to a global symmetry in the boundary conformal field theory. We would therefore most naturally be led to a model in which we gauge the global $U(2)$ symmetry of (4) and put it into an AdS Schwarzschild background. In order to trigger spontaneous symmetry breaking we introduce a chemical potential via a boundary value for the temporal component of the overall, Abelian $U(1)$ gauge field. This is then our gauged model.

Alternatively we might ask what are the minimal ingredients necessary to trigger spontaneous symmetry breaking. The chemical potential resides entirely in the overall $U(1)$ factor. The other three $S U(2)$ gauge fields are not needed to achieve symmetry breaking. Therefore 


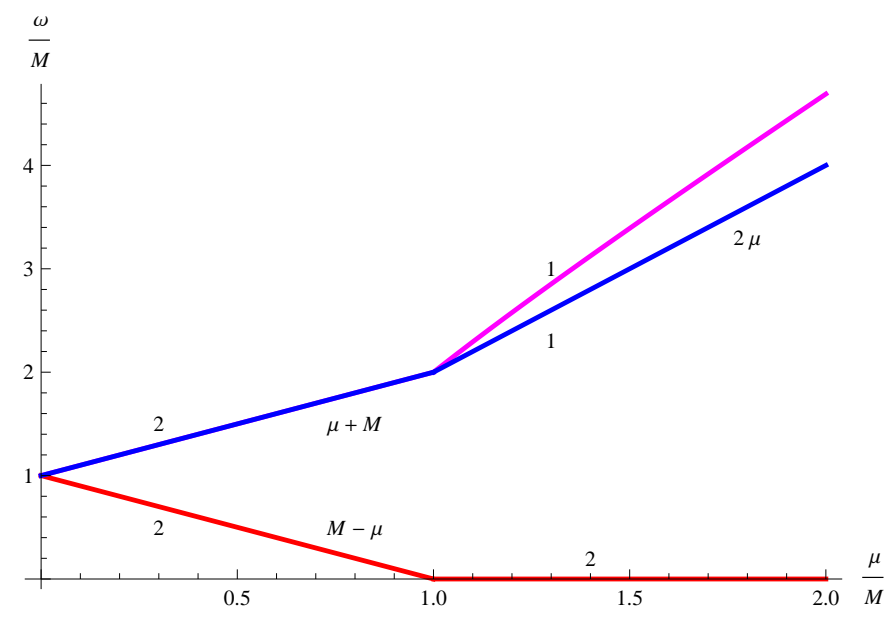

Figure 1: The spectrum of the field theoretical model. Below the critical value $\mu=M$ there are four massive modes. The masses are $M-\mu$ and $M+\mu$, the numbers indicate that they are doubly degenerate. In the broken phase $\mu>M$ there are two Goldstone modes with exactly zero mass and two gapped modes. The special gapped mode has mass $2 \mu$.

we can choose as a sort of minimal setup a model in which the $S U(2)$ symmetry stays global in the bulk of AdS. As already mentioned in the introduction this is a somewhat unusual realization of the symmetry from the boundary conformal field theory point of view. There are no conserved currents associated to this $S U(2)$ symmetry, nevertheless all states and operators fall naturally into representations of this symmetry group since it is a global symmetry of the bulk and it is also not broken by any of the boundary conditions. This setup constitutes our ungauged model and we will study it in detail in the next section.

Let us note here one more technical detail: the field theoretic model of this section is most naturally viewed as living in four space time dimensions. In the following our holographic models will be dual to field theories living in three space time dimensions in order to stay as close as possible to the well-studied holographic $U(1)$ s-wave superfluid of [2, 15. This is however of no relevance to the essential features of the models, i.e. the existence and the nature of the hydrodynamic and Goldstone modes.

\section{The ungauged model}

We will now study the holographic model where the condensation of a charged scalar breaks a global $S U(2)$ symmetry in the bulk. We shall look at the spectrum of quasinormal modes on both sides of the phase transition and study their dispersion relations. Since the simple $U(1)$ s-wave holographic superfluid constitutes a subsector of this as well as of the gauged model we will also use the opportunity to briefly review the most salient features of its QNM spectrum.

The minimal holographic model containing a type II Goldstone boson consists of a scalar 
doublet of $S U(2)$ charged under a $U(1)$ gauge field. The Lagrangian is given by

$$
\mathcal{L}=\left(-\frac{1}{4} F^{\mu \nu} F_{\mu \nu}-m^{2} \Psi^{\dagger} \Psi-\left(D^{\mu} \Psi\right)^{\dagger} D_{\mu} \Psi\right),
$$

where

$$
\Psi=\left(\begin{array}{c}
\lambda \\
\psi
\end{array}\right), \quad D_{\mu}=\partial_{\mu}-i A_{\mu}
$$

and $A_{\mu}$ is the Abelian gauge field. The mass of the scalar field is chosen to be $m^{2}=-2 / L^{2}$. This is basically the same as the model in [2] except that we have added a second scalar field $\lambda$ with the same mass. Because of the degeneracy in the mass the model possesses in addition to the bulk-local $U(1)$ symmetry a bulk-global $S U(2)$ symmetry. Note that the global $S U(2)$ symmetry is a priori not enough to set the field $\lambda(r)=0$. But we are interested in un-sourced static solutions for the scalar fields, i.e. we assume that the leading non-normalizable mode is not switched on. The solution space is then a two dimensional complex vector space spanned by the vevs of the operators dual to the scalar fields. On this parameter space we can act with the global $S U(2)$ symmetry to set the operator corresponding to the field $\lambda$ equal to zero. Since now the non-normalizable and the normalizable mode of $\lambda$ are set to zero it follows that $\lambda(r)=0$.

We will be working in the probe limit, in which the coupling of the gauge field is very large and the backreaction of the matter fields onto the metric can be neglected. The background metric is then taken to be the Schwarzschild-AdS black brane

$$
\begin{array}{r}
d s^{2}=-f(r) d t^{2}+\frac{d r^{2}}{f(r)}+\frac{r^{2}}{L^{2}}\left(d x^{2}+d y^{2}\right), \\
f(r)=\frac{r^{2}}{L^{2}}-\frac{M}{r} .
\end{array}
$$

The horizon of the black hole is located at $r_{H}=M^{1 / 3} L^{2 / 3}$ and its Hawking temperature is $T=\frac{3 r_{H}}{4 \pi L^{2}}$. In the following we use dimensionless coordinates

$$
(r, t, x, y) \rightarrow\left(r_{H} \rho, \frac{L^{2}}{r_{H}} \bar{t}, \frac{L^{2}}{r_{H}} \bar{x}, \frac{L^{2}}{r_{H}} \bar{y}\right) .
$$

These rescalings allow us to set $M=r_{H}=1$ in the dimensionless system. In order to switch on a finite chemical potential in the boundary theory, the bulk Maxwell field

$$
A=\chi(\rho) d \bar{t}
$$

must take a non-zero value at the boundary.

The equations of motion for the background fields are

$$
\begin{aligned}
& \chi^{\prime \prime}+\frac{2}{\rho} \chi^{\prime}-\frac{2 \psi^{2}}{f} \chi=0 \\
& \psi^{\prime \prime}+\left(\frac{f^{\prime}}{f}+\frac{2}{\rho}\right) \psi^{\prime}+\frac{\chi^{2}}{f^{2}} \psi-\frac{m^{2}}{f} \psi=0 .
\end{aligned}
$$


Notice that the system above is precisely the original $U(1)$ holographic superconductor first studied in [2]. To ensure finiteness of the norm of the current at the horizon, we have to demand the scalar field to be regular whereas the gauge field has to vanish $\chi(\rho=1)=0$. With these boundary conditions, the asymptotic behavior of the fields at the conformal boundary is

$$
\begin{gathered}
\chi=\bar{\mu}-\frac{\bar{n}}{\rho}+O\left(\frac{1}{\rho^{2}}\right), \\
\psi=\frac{\psi_{1}}{\rho}+\frac{\psi_{2}}{\rho^{2}}+O\left(\frac{1}{\rho^{3}}\right) .
\end{gathered}
$$

For the chosen value of the scalar mass, both terms in the scalar asymptotics correspond to normalizable modes [32]. Considering one or the other as the vacuum expectation value of a dual boundary operator leads to two different theories. In what follows we will consider only the case in which $\psi_{1}$ is interpreted as the coupling and $\psi_{2}$ as the vev of a mass dimension two operator.

The dimensionless parameters are related with the physical quantities by

$$
\begin{aligned}
\bar{\mu} & =\frac{3}{4 \pi T} \mu, \\
\bar{n} & =\frac{9}{16 \pi^{2} T^{2} L^{2}} n, \\
\psi_{1} & =\frac{3}{4 \pi T L^{2}} J_{\mathcal{O}}, \\
\psi_{2} & =\frac{9}{16 \pi^{2} T^{2} L^{4}}\langle\mathcal{O}\rangle,
\end{aligned}
$$

where $\mu, n$ and $J_{\mathcal{O}},\langle\mathcal{O}\rangle$ are the chemical potential, charge density and source and expectation value of an operator $\mathcal{O}$ of dimension 2, respectively. From now on we set $L=1$. In the following we will work in the grand canonical ensemble. In practice we vary the dimensionless parameter $\bar{\mu}$. Because of the underlying conformal symmetry this can then be thought of as either fixing the chemical potential $\mu$ and varying the temperature $T$ or fixing the temperature and varying the chemical potential. We define the temperature by $T / T_{c}=\bar{\mu}_{c} / \bar{\mu}$ and fix $\mu=1$.

Spontaneous symmetry breaking is driven by low temperature or high chemical potential. It triggers a non trivial expectation value for the scalar field without switching on any source $J_{\mathcal{O}}$. For small $\bar{\mu}$ the scalar field is trivial and the gauge equation is solved by $\chi=\bar{\mu}(1-1 / \rho)$ and $\psi=0$. The system is then in the symmetric phase. However, by decreasing the temperature the system becomes unstable towards condensation of the scalar [1, 2]. In [15] it was shown that at the critical temperature indeed the lowest quasinormal mode of the scalar field becomes unstable, i. e. it crosses over to the upper half plane.

The free energy density of the system is given by the on-shell renormalized action,

$$
F=-T S_{r e n}=-T\left(\frac{1}{2} \mu n-\int_{r_{H}}^{\infty} d r \frac{r^{2} \psi^{2} \chi^{2}}{f}\right)
$$


The second term vanishes in the absence of a condensate and it works against the phase transition if it is present. In Figure 2 the free energies for the symmetric and broken phase are compared. It is clear that for $T<T_{c}$ the condensate solution is always preferred and therefore the system undergoes a second order phase transition to the superconducting phase. Note that the presence of the second scalar plays no role for the phase structure. It simply vanishes in the broken and unbroken phase $\lambda=0$.
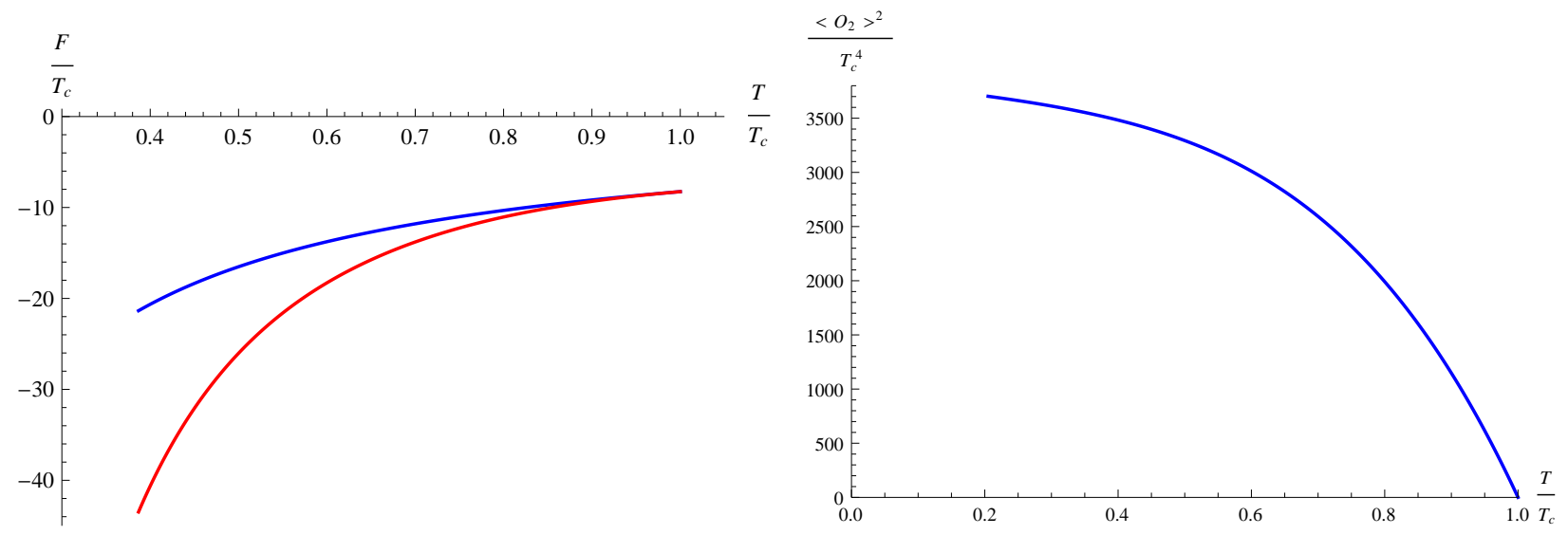

Figure 2: (Left) The free energy of the trivial (blue) and condensate (red) background solutions at low temperatures, $T<T_{c}$. (Right) Value of the condensate in the grand canonical ensemble as a function of $T / T_{c}$.

In order to extract the quasinormal mode spectrum, we switch on fluctuations of the background fields

$$
\begin{aligned}
\Psi^{\mathrm{T}} & =(\eta(\rho, t, x), \psi(\rho)+\sigma(\rho, t, x)) \\
A & =\left(\chi(\rho)+a_{t}(\rho, t, x)\right) d t+a_{x}(\rho, t, x) d x .
\end{aligned}
$$

We do not include transverse fluctuations because they decouple from the interesting physical features of the model at hand.

In the normal phase, i.e. expanding around $\psi(\rho)=0$, the system reduces to the $U(1)$ holographic superconductor studied in [15] with two copies of the scalar fluctuations,

$$
\begin{aligned}
f s^{\prime \prime}+s^{\prime}\left(f^{\prime}+\frac{2 f}{\rho}\right)+\left(\frac{(\chi+\omega)^{2}}{f}-\frac{k^{2}}{\rho^{2}}-m^{2}\right) s & =0, \\
f a_{t}^{\prime \prime}+\frac{2 f}{\rho} a_{t}^{\prime}-\frac{k^{2}}{\rho^{2}} a_{t}-\frac{\omega k}{\rho^{2}} a_{x} & =0, \\
f a_{x}^{\prime \prime}+f^{\prime} a_{x}^{\prime}+\frac{\omega^{2}}{f} a_{x}+\frac{\omega k}{f} a_{t} & =0, \\
\frac{i \omega}{f} a_{t}^{\prime}+\frac{i k}{\rho^{2}} a_{x}^{\prime} & =0,
\end{aligned}
$$


where $s$ stands for both $\eta$ and $\sigma$ fluctuations. The equation for the complex conjugate scalar $\bar{s}$ can be obtained by changing the sign of the potential $\chi$ in (30). The frequency and momentum are related to the physical ones by

$$
\begin{aligned}
\omega & =\frac{3}{4 \pi T} \omega_{p h}, \\
k & =\frac{3}{4 \pi T} k_{p h} .
\end{aligned}
$$

The scalar and gauge fluctuations completely decouple in the symmetric phase. This is a consequence of working in the probe limit. The quasinormal mode spectrum of the $U(1)$ field in the normal phase is just that of an electromagnetic field on an AdS-Sch background. The longitudinal fluctuations contain one hydrodynamic mode, $\omega=-i D k^{2}$, reflecting the diffusive behavior of normal fluids. In physical units $D=3 /(4 \pi T)$. Due to the lack of an energy-momentum tensor for the dual field theory in the probe limit, the diffusion pole is the only hydrodynamic mode in the unbroken phase.

There are two copies of the scalar fluctuations. The quasinormal modes of $\eta$ and $\sigma$ move towards the origin when decreasing the temperature, whereas the modes of $\bar{\eta}$ and $\bar{\sigma}$ have larger masses and widths the smaller the temperature. As we approach the critical temperature $T=T_{c}$, the lowest quasinormal modes of $\eta$ and of $\sigma$ become massless, triggering the phase transition: the scalar field acquires a non trivial vev in order to avoid its fluctuations to become tachyonic. By symmetry we can choose the condensate to reside completely in the $\psi$ field. The fluctuations $\sigma$ couple then to the gauge field fluctuations just as in [15]. Therefore the QNM spectrum in this sector contains a Goldstone mode with linear dispersion relation $\omega= \pm v_{s} k+O\left(k^{2}\right)$. This is the usual type I Goldstone boson associated with the breaking of the gauge $U(1)$ symmetry. As shown in [15] it can be interpreted as the sound mode of the dual superfluid in the broken phase. What happens then to the QNMs in the fluctuations of the second scalar $\eta$ ? At the critical temperature there is also an ungapped mode present since its QNM spectrum is simply another copy of the scalar sector. Since there are no operators generating the $S U(2)$ symmetry in the dual field theory standard arguments about the appearance of Goldstone modes do a priori not apply. Three logical possibilities arise then: the mode could become unstable for $T<T_{c}$, it could become gapped again or it stays ungapped, playing the role of an unexpected Goldstone boson for the broken bulk-global $S U(2)$ symmetry. Shortly we will see that the last possibility is realized and that the massless mode of $\eta$ will indeed correspond to a type II Goldstone boson with quadratic dispersion relation, $\omega \propto k^{2}$. 
In the broken phase, the equations of motion read

$$
\begin{aligned}
& 0=f \eta^{\prime \prime}+\eta^{\prime}\left(f^{\prime}+\frac{2 f}{\rho}\right)+\left(\frac{(\chi+\omega)^{2}}{f}-\frac{k^{2}}{\rho^{2}}-m^{2}\right) \eta \\
& 0=f \delta^{\prime \prime}+\delta^{\prime}\left(f^{\prime}+\frac{2 f}{\rho}\right)+\left(\frac{\chi^{2}}{f}+\frac{\omega^{2}}{f}-\frac{k^{2}}{\rho^{2}}-m^{2}\right) \delta-\frac{2 i \omega \chi}{f} \zeta-i \psi\left(\frac{\omega}{f} a_{t}+\frac{k}{\rho^{2}} a_{x}\right) \\
& 0=f \zeta^{\prime \prime}+\zeta^{\prime}\left(f^{\prime}+\frac{2 f}{r}\right)+\left(\frac{\chi^{2}}{f}+\frac{\omega^{2}}{f}-\frac{k^{2} L^{2}}{r^{2}}-m^{2}\right) \zeta+\frac{2 i \omega \chi}{f} \delta+\frac{2 \chi \psi}{f} a_{t} \\
& 0=f a_{t}^{\prime \prime}+\frac{2 f}{\rho} a_{t}^{\prime}-\left(\frac{k^{2}}{\rho^{2}}+2 \psi^{2}\right) a_{t}-\frac{\omega k}{\rho^{2}} a_{x}-2 i \omega \psi \delta-4 \chi \psi \zeta \\
& 0=f a_{x}^{\prime \prime}+f^{\prime} a_{x}^{\prime}+\left(\frac{\omega^{2}}{f}-2 \psi\right) a_{x}+\frac{\omega k}{f} a_{t}+2 i k \psi \delta \\
& 0=\frac{i \omega}{f} a_{t}^{\prime}+\frac{i k}{\rho^{2}} a_{x}^{\prime}+2 \psi^{\prime} \delta-2 \psi \delta^{\prime}
\end{aligned}
$$

where we have divided $\sigma=\zeta+i \delta$ into real and imaginary part. The system (37)-(41) is again the one studied in [15]. This sector, that also appears in the gauged model that will be presented afterwards, decouples from the additional scalar fluctuation $\eta$. Notice that even if (36) is formally the same as in the normal phase, the background $\chi$ is different leading to non trivial features in the $\eta$ sector such as the presence of a massless excitation.
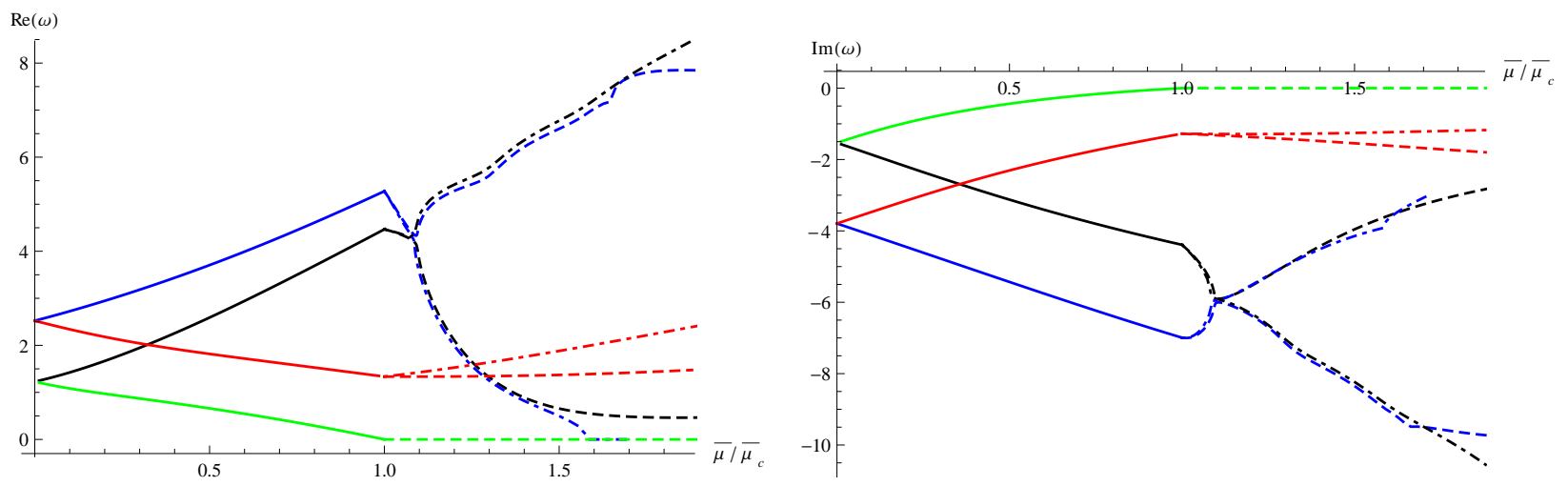

Figure 3: Real (left) and imaginary (right) parts of the lowest scalar QNMs as a function of the chemical potential. Solid lines correspond to the unbroken phase. For the broken phase dashed lines stand for modes of the additional scalar while dotdashed lines represent the modes common to the $U(1)$ holographic superconductor.

Figure 3 shows the spectrum of quasinormal excitations of the scalar doublet. In the normal phase we have two degenerate copies of the spectrum that partially split after the phase transition. It is clear that the two lowest excitations become massless at the critical chemical potential and then remain massless in the superconducting phase. They can be identified with the two Goldstone bosons at the phase transition. The rest of the excitations 
remain gapped in the broken phase. Notice that the first $\bar{\eta}$ excitation (dashed black line in figure 3) does not follow the expected universal behavior in the broken phase, i.e. it is not linear in $\mu$. This mode is the equivalent of the special gapped mode $\omega_{4}$ in the field theoretical model of section 2. However, it has already been mentioned that the ungauged model does not satisfy all the theorems about symmetry breaking and therefore deviations from the universal behavior should not be surprising. The behavior of the gapped modes is actually similar to that of the $U(1)$ model modes. In the unbroken phase we can distinguish the modes that come from the $s$-type of fluctuations from the ones that come from the complex conjugate $\bar{s}$ fluctuations. The former become lighter whereas the latter become heavier ${ }^{5}$. In the broken phase it is more useful to use real and imaginary parts, at least for the scalar that mixes with the gauge fields fluctuations, i.e. the lower component of the scalar in our conventions. So we can not a priori talk of $s$ and $\bar{s}$ type fluctuations. We still can study to which modes the $s$ and $\bar{s}$ type modes connect to in the broken phase. Here we see an interesting pattern: the $s$ type modes split in the broken phase whereas the $\bar{s}$ type modes stay almost degenerate close to the phase transition (at least at zero momentum). This is surprising given the fact that the fluctuations correspond to two completely different systems, one coming from a single differential equation whereas the others come from a complicated system of coupled equations. However, for small temperatures they split and actually the real part of the lowest one for the $U(1)$ sector goes to zero at a finite temperature. For temperatures below $T \approx 0.63 T_{c}$ it becomes a purely imaginary mode.

Sound mode: There are two massless modes in the broken phase. The first one is the type I Goldstone boson appearing because of the spontaneous breaking of the $U(1)$ gauge symmetry. In [15], it was shown that this mode corresponds to the sound mode of the dual superfluid and that in the hydrodynamic limit it has a linear dispersion relation

$$
\omega_{I}= \pm\left(v_{s} k+\bar{b} k^{2}\right)-i \Gamma_{s} k^{2}
$$

where $v_{s}$ is the speed of sound and $\Gamma_{s}$ is its attenuation. It turns out that the quadratic part of the dispersion relation also has a real component. This component is very small and subleading compared to the linear term that determines the speed of sound. In [15] this real quadratic part has not been studied.

For very small temperatures the velocity approaches its conformal value $v_{s}^{2}=1 / 2$ while the width goes to zero, see figure 4 . Close to the phase transition, the speed of sound has a mean field behavior as a function of temperature

$$
v_{s}^{2} \approx 2.8\left(1-\frac{T}{T_{c}}\right)
$$

As expected, at $T=T_{c}$ the speed of sound vanishes. This can be traced back to the fact that at the phase transition the total mass $m_{*}^{2}=M^{2}-\mu^{2}$ fulfills $m_{*}^{2}=v^{2}=0$, as expected, and hence the complex scalar field, charged under a $U(1)$ symmetry, becomes massless.

\footnotetext{
${ }^{5}$ This behavior is reversed if we had taken the chemical potential to be negative.
} 

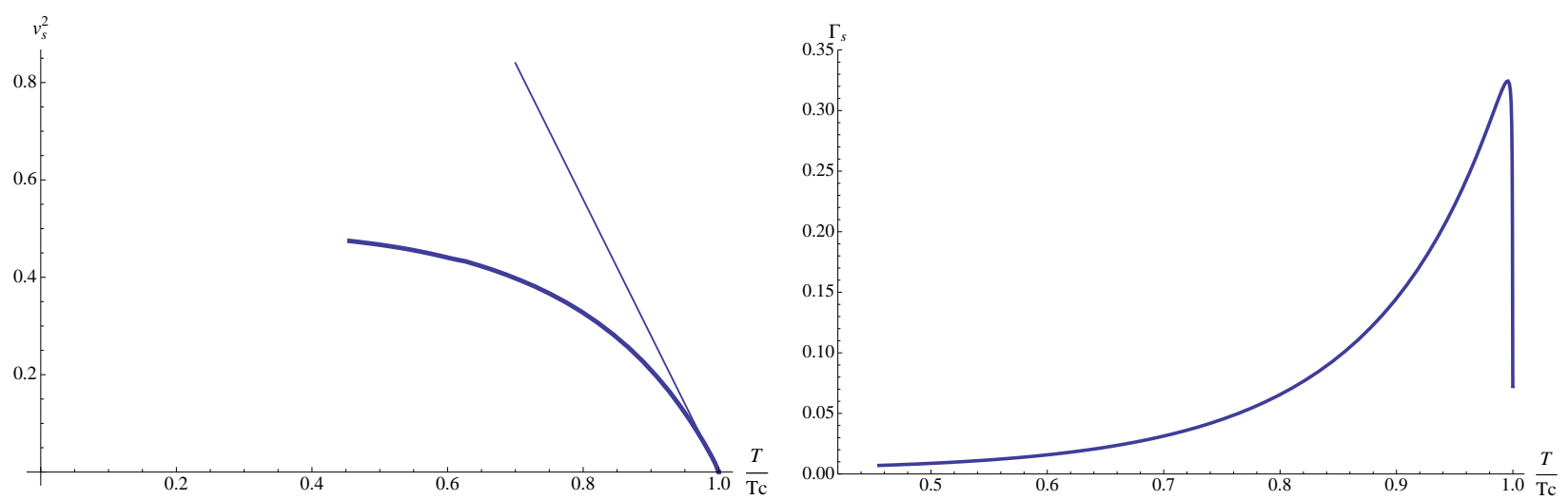

Figure 4: Speed of sound and damping for the sound mode. The speed of sound goes to zero at the critical temperature. The damping constant first rises quickly and then falls off again. Precisely at the critical temperature its value is such that the sound modes connect continuously to the scalar modes that become massless there. The peak in the damping constant sits close to the critical temperature and was not resolved in [15].

Indeed, one can write down the effective action, analogous to (4), for a complex scalar field with mass $M$, in the presence of a chemical potential for a $U(1)$ symmetry that is spontaneously broken. The excitations on top of the $U(1)$-breaking background have a dispersion relation equal to (8)-(9), being (8) the type I Goldstone boson. It is a general feature of these linear sigma models that the coefficient in front of the linear term in the momentum depends on $m_{*}^{2}$, as can be explicitly checked for the case at hand (see (8)). Therefore, at the phase transition the leading term in the dispersion relation is of $O\left(k^{2}\right)$; this effect can be seen very clearly with numerical methods, as shown in Figure 5. Since the quasinormal mode spectrum has to vary continuously through the second order phase transition the real and complex coefficients of the $k^{2}$ term have to coincide at $T=T_{c}$ with the ones obtained from the massless scalars in the unbroken phase. Numerically we find $\bar{b}\left(T_{c}\right)=0.22$ and $\Gamma_{s}\left(T_{c}\right)=0.071$.

Pseudo diffusion mode: In the unbroken phase our model has only one hydrodynamic mode, the diffusion mode $\omega=-i D k^{2}+O\left(k^{4}\right)$ with $D=3 /(4 \pi T)$ in physical units. The shear and normal sound modes have their origin in the metric fluctuations and therefore are absent in the decoupling limit we are studying. The phase transition to the broken phase is second order. For the spectrum of quasinormal modes this implies that the modes of the broken and unbroken phase must connect continuously through the phase transition. In the case of the diffusion mode there must therefore exist a quasinormal mode with purely imaginary frequency. Hydrodynamics implies however that the only ungapped modes are the sound modes corresponding to the type I Goldstone mode. Not too far from the phase transition, i.e. for $T \lesssim T_{c}$ the diffusion mode of the broken phase must develop into a mode with dispersion relation

$$
\omega=-i \gamma(T)-i D(T) k^{2}+O\left(k^{4}\right)
$$




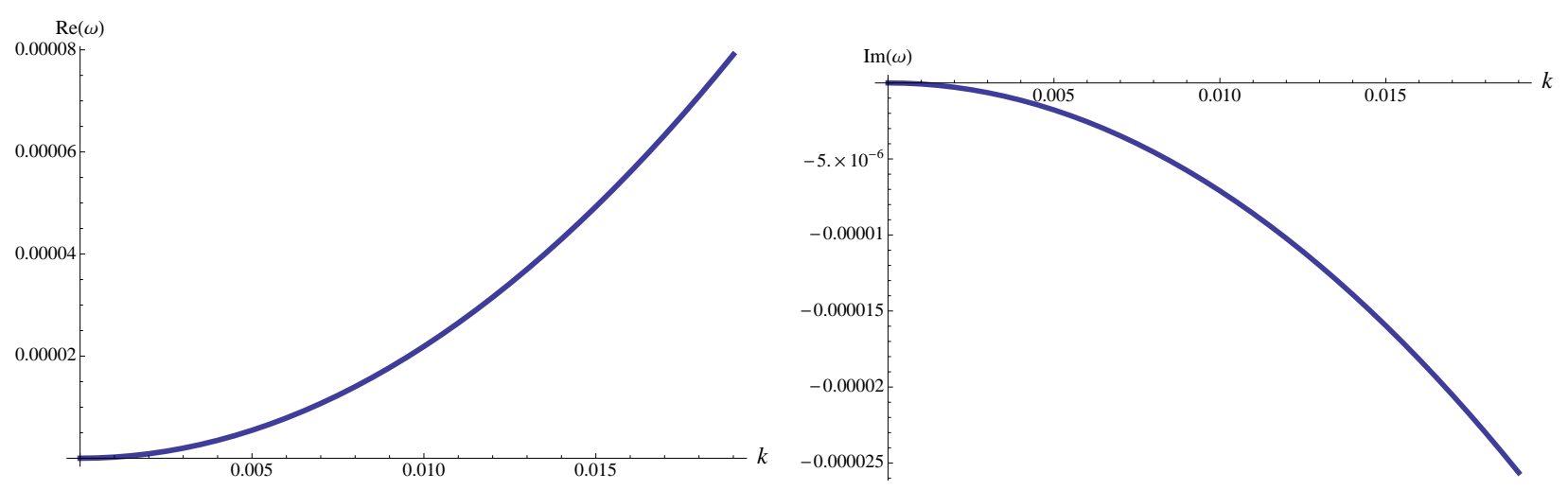

Figure 5: Dispersion relations of $\operatorname{Re} \omega$ (left) and $\operatorname{Im} \omega$ (right) at $T=T_{c}$ for the type I Goldstone boson in the system studied by [15]. The behavior Re $\omega \sim k$ becomes quadratic right at this temperature: $\operatorname{Re} \omega=\bar{b} k^{2}$. The coefficient is $\bar{b}=0.22$, which in turn matches the value that one finds if approaches $T_{c}$ from above (i.e. from the unbroken phase).

as shown in Figure 6.

We might say that the diffusion mode develops a gap in the broken phase and becomes what has been called a pseudo diffusion mode in [15]. Precisely at zero momentum $k=0$ this gapped pseudo diffusion mode explains the observation made in [17] on the late time response of holographic superconductors. For temperatures $T \lesssim T_{c}$ the pseudo diffusion mode is the mode that lies closest to the real axis and therefore it dominates the long time response to any perturbation, such as the quenches studied in [17]. It follows that the order parameter shows a purely exponential decay since this mode does not have a real frequency. The existence of that mode can ultimately be traced back to the universality of the diffusion mode in the unbroken phase. We expect therefore the pseudo diffusion mode to be a universal feature of a wide class of superfluids (not necessarily holographic ones).

The gap $\gamma$ grows as the temperature decreases. On the other hand there are quasinormal modes (connecting to the QNMs in the scalar sector of the unbroken phase) whose imaginary part is only weakly dependent on the temperature. At a certain crossover temperature $T_{*}$ the gap of the pseudo diffusion mode is bigger than the imaginary part of these modes, as shown in Figure 7. Then the response pattern changes from a purely exponential decay to an exponentially damped oscillation. Numerically we find that the crossover temperature is $T_{*}=0.69 T_{c}{ }^{6}$ Such crossover changes in the long term response appear frequently in the details of the quasinormal mode spectrum of holographic field theories, [33, 34, 35]. In fact this purely exponential decay applies not only to the order parameter but to all operators that correspond to the fields participating in the fluctuation system (37)-(41), e.g. charge density or $x$-component of the current.

For finite momentum the response pattern is expected to be different however. Now one also has to take into account the sound mode. While precisely at zero momentum the sound

\footnotetext{
${ }^{6}$ This is lower than in the model of [17. The difference is presumably due to the fact that we work in the decoupling limit.
} 

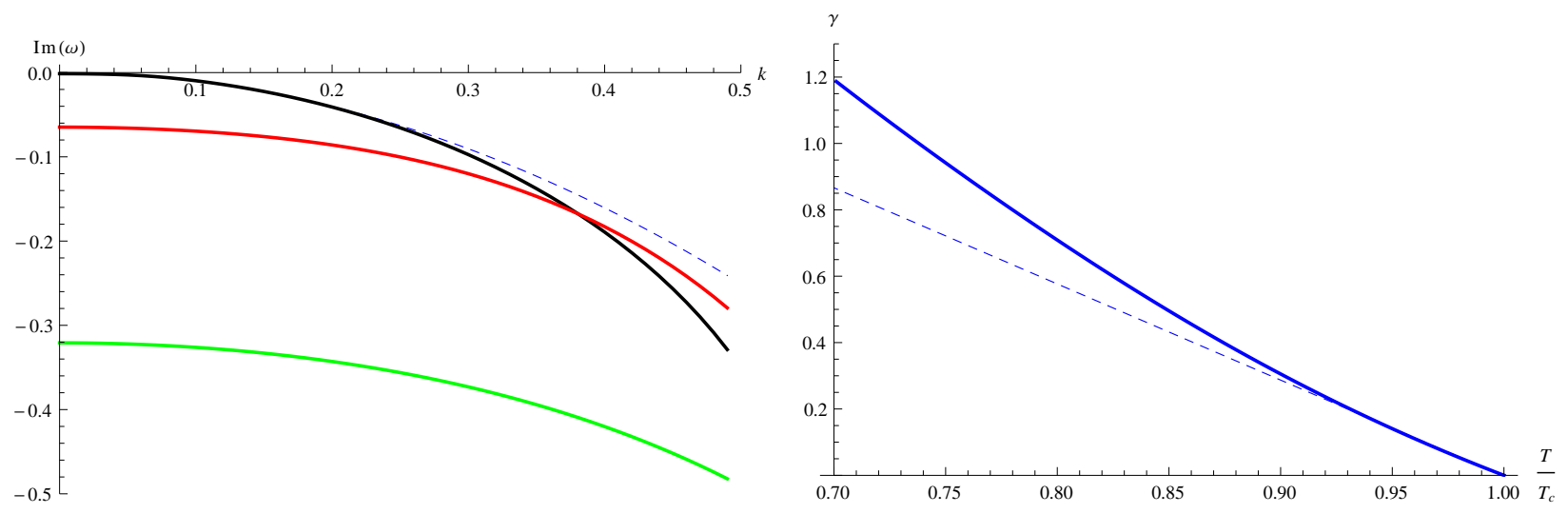

Figure 6: (Left) Dispersion relation of the gapped pseudo diffusion mode in the broken phase for three different temperatures. The gap widens as the temperature is lowered. (Right) Gap $\gamma$ as a function of the reduced temperature $T / T_{c}$. As one approaches the critical temperature from below the gap vanishes linearly.
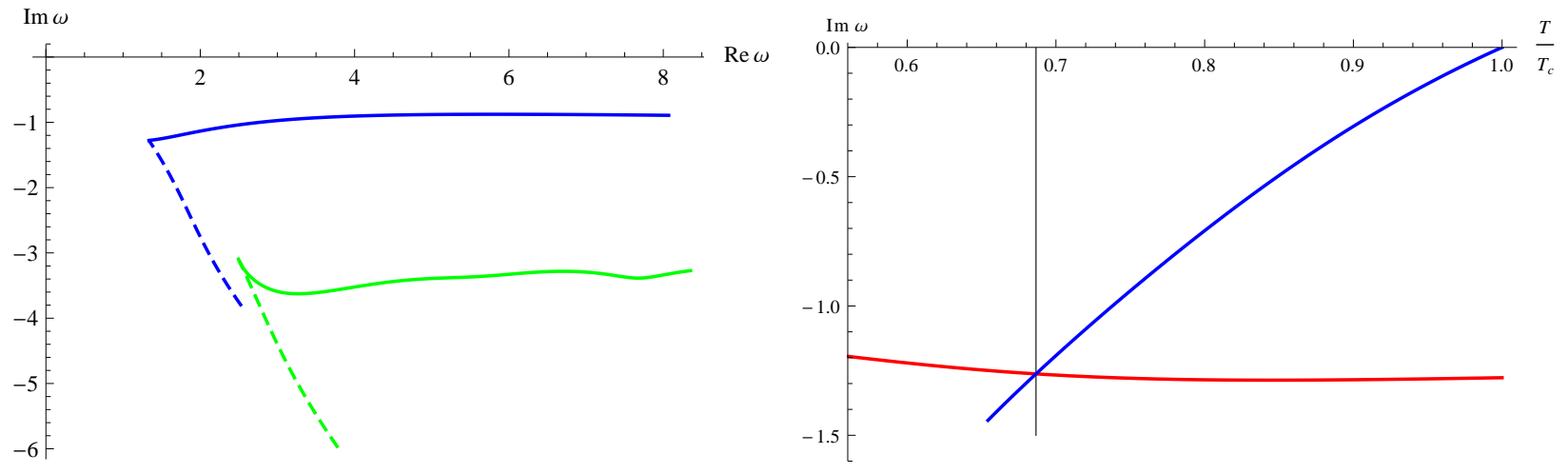

Figure 7: (Left) Continuation of the second and third scalar QNM into the broken phase. The real part grows as the temperature is lowered whereas the imaginary part shows very little dependence on $T$. (Right) The gap $\gamma$ (blue line) and the imaginary part of the lowest (scalar) mode fluctuation (red line) in the broken phase are shown as function of $T / T_{c}$. At $T_{*} \approx 0.69 T_{c}$ the imaginary parts cross. For lower temperatures the late time response is not dominated anymore by the pseudo diffusion mode and consequently is in form of a exponentially decaying oscillation. 
mode, i.e. the Goldstone mode, degenerates to a constant phase change of the condensate at small but non-zero momentum the long time response should be dominated by the complex frequencies (42). If one looks however only to the response in the gauge invariant order parameter $|\mathcal{O}|$ the Goldstone modes, being local phase rotations of the order parameter, are projected out.

Type II Goldstone mode: The second massless mode is the Goldstone boson associated with the breaking of the bulk-global $S U(2)$ symmetry. It can be fit to a quadratic dispersion relation of the form

$$
\omega_{I I}= \pm b k^{2}-i c k^{2}+O\left(k^{4}\right),
$$

in the long wavelength limit. Therefore it has the characteristic of a type II Goldstone mode. In Figure 8 the dispersion relation for the $\eta$ massless mode is shown for various temperatures as well as its fit to the hydrodynamic form. It is clear that there is a good agreement in the regime of validity of the low energy limit.
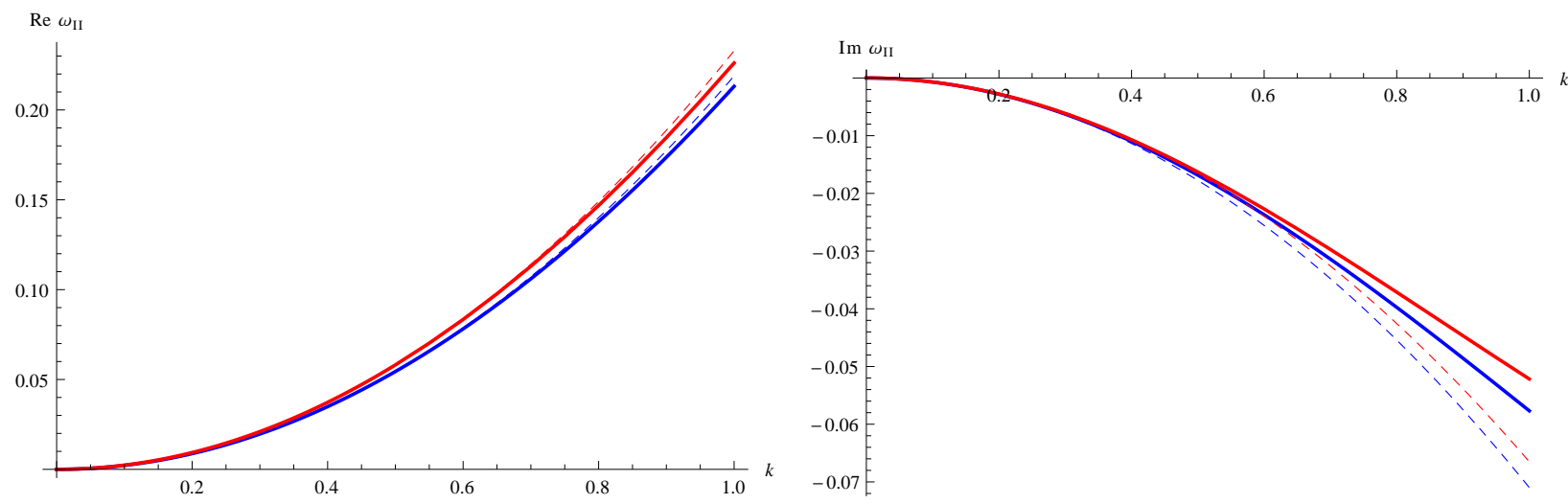

Figure 8: Real (left) and imaginary (right) parts of the type II Goldstone mode as a function of the momentum for $T / T_{c}=0.9998$ (blue) and $T / T_{c}=0.704$ (red). The solid lines correspond to the numerical result while the dashed lines are the quadratic fit to a dispersion relation $\omega_{I I}=b k^{2}-i c k^{2}$.

The coefficients in the hydrodynamic dispersion relation 45 as a function of the temperature are shown in Figure 9. Close to the phase transition they have a linear dependence in the reduced temperature

$$
\begin{aligned}
& b(T)=0.22+0.049\left(1-\frac{T}{T_{c}}\right), \\
& c(T)=0.071-0.0014\left(1-\frac{T}{T_{c}}\right) \text { near } T_{c} .
\end{aligned}
$$

Notice that at the phase transition the sound mode and the type II Goldstone must behave in the same way due to continuity of the modes through the phase transition and the fact that they are degenerate in the normal phase. In fact, at the transition $b=\bar{b}=0.22$ and 
$c=\Gamma_{s}=0.071$, values that of course coincide with those of the lowest scalar mode in the normal phase. On the other hand, it is interesting to notice that in the broken phase the behavior of the coefficients of the type II Goldstone is completely different from that of the coefficients of the sound of the superfluid. Unlike the sound velocity, that vanishes at the phase transition, the coefficient $b$ of the type II Goldstone mode takes a finite value at the critical temperature. This result of course persists for the gauged model. The attenuation on the other hand, as it happens for the $U(1)$ sector, has a finite value at the phase transition and then decreases with temperature, reflecting the fact that the fluid is more ideal the lower the temperature.
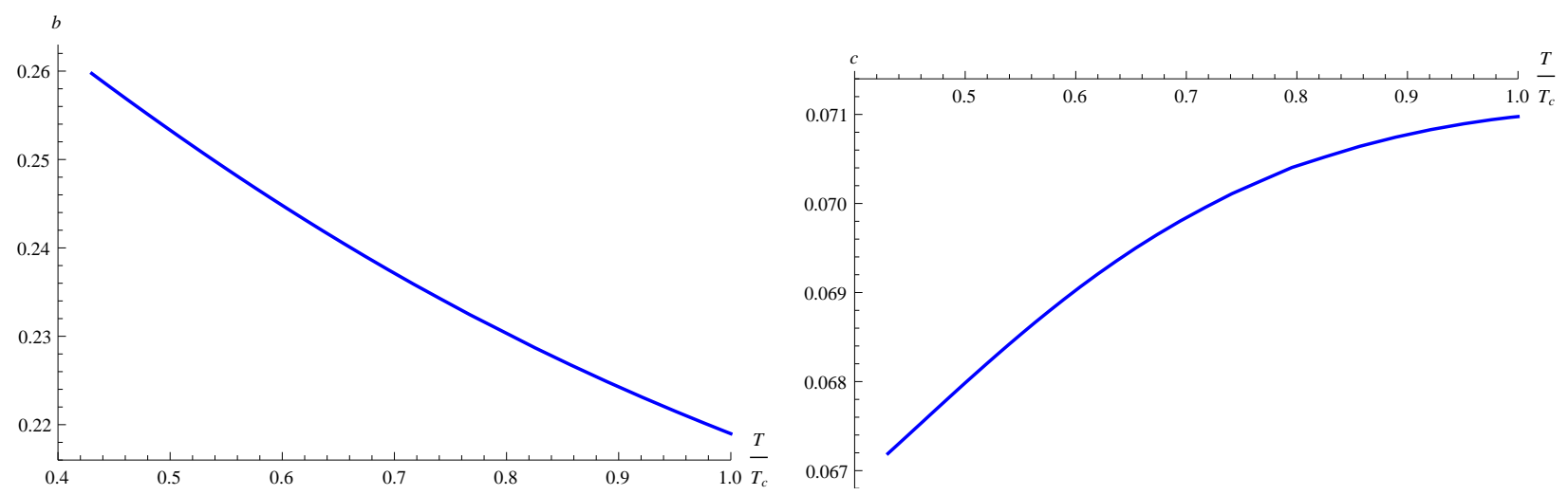

Figure 9: Coefficients of the type II Goldstone mode dispersion relation $\omega_{I I}=b k^{2}-i c k^{2}$, as a function of the temperature. Dependence with temperature is very mild.

\section{The gauged model}

Let us now discuss the fully gauged model. Consider the following Lagrangian for a complex scalar field living in the fundamental representation of a $U(2)$ gauge symmetry of the bulk,

$$
S=\int \sqrt{-g} \mathcal{L}=\int d^{4} x \sqrt{-g}\left(-\frac{1}{4} F^{\mu \nu c} F_{\mu \nu}^{c}-m^{2} \Psi^{\dagger} \Psi-\left(D^{\mu} \Psi\right)^{\dagger} D_{\mu} \Psi\right)
$$

where

$$
\Psi=\left(\begin{array}{c}
\lambda \\
\psi
\end{array}\right), \quad A_{\mu}=A_{\mu}^{c} T_{c}, \quad D_{\mu}=\partial_{\mu}-i A_{\mu}
$$

and $c=0,1,2,3$ is the color index. The field $\Psi$ plays the role of the condensate. The expectation value of its dual operator thus triggers the spontaneous breaking of the $U(2)$ global symmetry of the boundary theory. For simplicity, we set $\lambda=0$ in the background. $T_{c}$ 
are the generators of $U(2)$ :

$$
\begin{aligned}
& T_{0}=\frac{1}{2} \mathbb{I}, \quad T_{i}=\frac{1}{2} \sigma_{i}, \\
& \left\{T_{i}, T_{j}\right\}=\frac{1}{2} \delta_{i j} \mathbb{I}, \quad\left\{T_{0}, T_{i}\right\}=\frac{1}{2} \sigma_{i} .
\end{aligned}
$$

Notice that we are again working in the probe limit, so the background metric is taken to be the Schwarzschild-AdS black brane of (16). On the other hand, the gauge field is now

$$
A_{0}^{(0)} \equiv \Phi(r), \quad A_{0}^{(3)} \equiv \Theta(r) .
$$

The rest of the components of the gauge field being zero. As in the previous section, we will use dimensionless coordinates defined by the rescaling given in (17).

The equations of motion for our ansatz are

$$
\begin{aligned}
\psi^{\prime \prime}+\left(\frac{f^{\prime}}{f}+\frac{2}{\rho}\right) \psi^{\prime}+\frac{(\Phi-\Theta)^{2}}{4 f^{2}} \psi-\frac{m^{2}}{f} \psi & =0, \\
\Phi^{\prime \prime}+\frac{2}{\rho} \Phi^{\prime}-\frac{\psi^{2}}{2 f}(\Phi-\Theta) & =0, \\
\Theta^{\prime \prime}+\frac{2}{\rho} \Theta^{\prime}+\frac{\psi^{2}}{2 f}(\Phi-\Theta) & =0 .
\end{aligned}
$$

Notice that from (53) it follows that we can not simply switch on $\Phi$ without also allowing for a non-trivial $\Theta$. We are of course only interested in switching on a chemical potential in the overall $U(1)$, and therefore we will impose $\Theta(\rho \rightarrow \infty)=0$ and allow for a finite boundary value of $\Phi$.

The coupled system of equations above can be simplified by defining $\chi \equiv \frac{1}{2}(\Phi-\Theta)$ and $\xi \equiv \frac{1}{2}(\Phi+\Theta)$. Using $(52)$ and $(53)$, we see that the resulting equations for these fields are7

$$
\begin{aligned}
& \Psi^{\prime \prime}+\left(\frac{f^{\prime}}{f}+\frac{2}{\rho}\right) \Psi^{\prime}+\frac{\chi^{2}}{f^{2}} \Psi-\frac{m^{2}}{f} \Psi=0, \\
& \chi^{\prime \prime}+\frac{2}{\rho} \chi^{\prime}-\frac{2 \Psi^{2}}{f} \chi=0, \\
& \xi^{\prime \prime}+\frac{2}{\rho} \xi^{\prime}=0,
\end{aligned}
$$

where we have redefined $\psi \rightarrow \sqrt{2} \Psi$. As usual we choose the boundary conditions $\chi(\rho=1)=$ $0, \xi(\rho=1)=0$ along with regularity of $\Psi$. Having a dual field theory with only one finite chemical potential switched on, implies that $\chi$ and $\xi$ must take the same non trivial value at the boundary in order to ensure that $\Theta$ vanishes asymptotically. Notice that $\xi$ decouples

\footnotetext{
${ }^{7}$ These equations of motion correspond to the probe limit of the system studied in [36] as a dual of superconductors with chemical potential imbalance. Notice however that in 36] the gauge symmetry was $U(1) \times U(1)$ instead of $U(2)$ as in the present setup.
} 
completely. The remaining system (54)-(55) is again the background found for the widely studied s-wave $U(1)$ holographic superconductor. Therefore, the background of the $U(2)$ gauge model contains the Abelian superconductor plus a decoupled conserved $U(1)$ sector.

The field $\chi$ lies in the direction of one of the broken generators, which is the linear combination $\frac{1}{2}\left(T_{3}-T_{0}\right)$, whereas $\xi$ lies in the direction of the preserved $U(1)$ given by $\frac{1}{2}\left(T_{3}+T_{0}\right)$.

The asymptotic expansion of the fields near the conformal boundary reads

$$
\begin{gathered}
\chi=\bar{\mu}_{\chi}-\frac{\bar{n}_{\chi}}{\rho}+O\left(\frac{1}{\rho^{2}}\right), \\
\xi=\bar{\mu}_{\xi}-\frac{\bar{n}_{\xi}}{\rho}+O\left(\frac{1}{\rho^{2}}\right), \\
\Psi=\frac{\psi_{1}}{\rho}+\frac{\psi_{2}}{\rho^{2}}+O\left(\frac{1}{\rho^{3}}\right) .
\end{gathered}
$$

The map of the various coefficients in the previous equations to the boundary conditions is $\bar{\mu}_{\chi}=\bar{\mu}_{\xi}=\bar{\mu}$. We will again focus in the $\mathcal{O}_{2}$ theory exclusively, henceforth we will demand $\psi_{1}=0$.

Equations (54)-(55) allow for solutions with a non-vanishing condensate, and therefore $\frac{1}{2}\left(T_{3}-T_{0}\right)$ will be spontaneously broken. This solution must be found numerically, since the system is non-linear. However, (56) does have an analytic solution

$$
\xi=\bar{\mu}\left(1-\frac{1}{\rho}\right)
$$

and thus $\bar{n}_{\xi}=\bar{\mu}$.

When the symmetry is not broken, $\Psi=0$, the equation for $\chi$ has of course

$$
\chi=\bar{\mu}\left(1-\frac{1}{\rho}\right)
$$

as a solution as well. Therefore, in the unbroken phase

$$
\begin{aligned}
& \Theta=0, \\
& \Phi=2 \bar{\mu}\left(1-\frac{1}{\rho}\right) .
\end{aligned}
$$

This behavior reflects the fact that $T_{3}$ is completely independent from $T_{0}$ in the unbroken phase. However, once we switch on the condensate, the interplay between $T_{3}$ and $T_{0}$ (recall that the remaining symmetry is a combination of the two) makes it impossible to set only one of the fields to zero.

Finally, let us mention that in order to relate the dimensionless parameters with the physical ones, we need to apply the same dictionary (23)-(26) used for the ungauged model. 


\subsection{Charge Density in the broken phase}

According to [19, 24] we can expect the presence of type II Goldstone modes if the broken symmetry generators fulfill

$$
\left\langle\left[Q_{a}, Q_{b}\right]\right\rangle=B_{a b}
$$

with at least one $B_{a b} \neq 0$. In our case we have $\left[Q_{1}, Q_{2}\right]=i Q_{3}$. Therefore in the broken phase we are interested in a non-vanishing expectation value for the charge density operator $\left\langle Q_{3}\right\rangle=n_{\Theta}$. As we argued previously, in the unbroken phase we necessarily have $\Theta(r)=0$. This happened since both $\chi$ and $\xi$ obey the same differential equation and the integration constants had to be set equal in order to do not switch on a source for $\Theta$. Now we would like to find out whether or not an expectation value for $\Theta$ will be spontaneously generated in the broken phase.

Independently of the phase the field associated to the unbroken combination of generators is given by (60). Since $\Theta=\xi-\chi$, then

$$
\bar{n}_{\Theta}=\bar{\mu}-\bar{n}_{\chi} .
$$

Hence, what we want to check is the difference between the leading and the subleading coefficients of $\chi$ as a function of the temperature. The numerical result is shown in Figure 10.

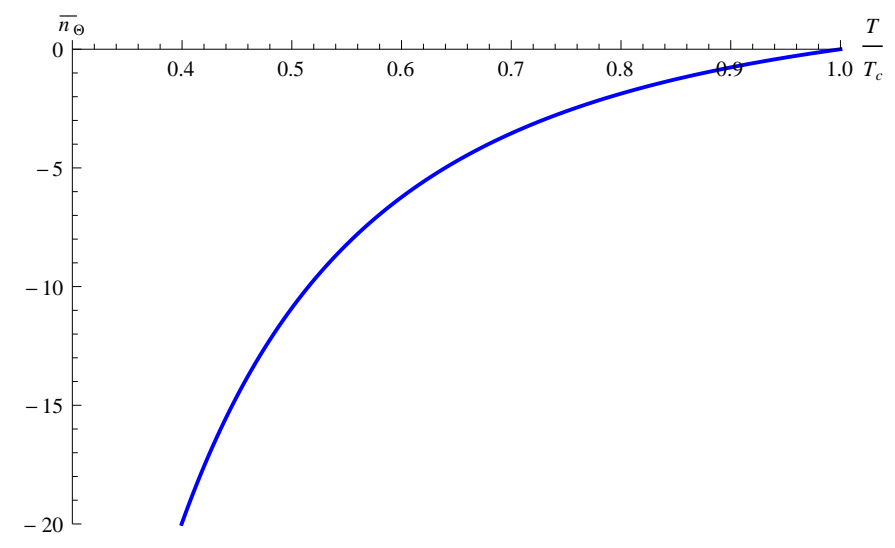

Figure 10: Charge density of $\Theta, \bar{n}_{\Theta}$, as a function of the temperature $T / T_{c}$.

So we conclude that precisely at $T \leq T_{c}$ this difference is switched on and an expectation value for $\left\langle Q_{3}\right\rangle$ appears. This can be taken as a clear indication for the appearance of type II Goldstone bosons in the spectrum. 


\subsection{Fluctuations of the gauged model}

In order to study the quasinormal spectrum and the conductivities of the system, we switch on longitudinal perturbations on top of the background, so that

$$
\begin{aligned}
\hat{\Psi}^{T} & =(\eta(t, \rho, x), \Psi(\rho)+\sigma(t, \rho, x)), \\
A^{(0)} & =\left(\Phi(\rho)+a_{t}^{(0)}(t, \rho, x)\right) d t+a_{x}^{(0)}(t, \rho, x) d x, \\
A^{(1)} & =a_{t}^{(1)}(t, \rho, x) d t+a_{x}^{(1)}(t, \rho, x) d x, \\
A^{(2)} & =a_{t}^{(2)}(t, \rho, x) d t+a_{x}^{(2)}(t, \rho, x) d x, \\
A^{(3)} & =\left(\Theta(\rho)+a_{t}^{(3)}(t, \rho, x)\right) d t+a_{x}^{(3)}(t, \rho, x) d x .
\end{aligned}
$$

\subsubsection{Perturbations in the Unbroken Phase}

In the normal phase, the background value of the condensate vanishes. Moreover, we have $\Theta(\rho)=0$. The equations of motion for the perturbations read

$$
\begin{aligned}
s^{\prime \prime}+s^{\prime}\left(\frac{f^{\prime}}{f}+\frac{2}{\rho}\right)+\left(\frac{\left(\frac{\Phi}{2}+\omega\right)^{2}}{f^{2}}-\frac{k^{2}}{f \rho^{2}}-\frac{m^{2}}{f}\right) s & =0, \\
a_{t}^{\prime \prime(c)}+\frac{2}{\rho} a_{t}^{\prime(c)}-\frac{\omega k}{f \rho^{2}} a_{x}^{(c)}-\frac{k^{2}}{f \rho^{2}} a_{t}^{(c)} & =0, \\
a_{x}^{\prime \prime(c)}+\frac{f^{\prime}}{f} a_{x}^{\prime(c)}+\frac{\omega^{2}}{f^{2}} a_{x}^{(c)}+\frac{\omega k}{f^{2}} a_{t}^{(c)} & =0, \\
\frac{\omega}{f} a_{t}^{(c)}+\frac{k}{\rho^{2}} a_{x}^{(c)} & =0,
\end{aligned}
$$

where $s \in\{\eta, \sigma\}$. Since the color indices do not see each other the system is the same one as (30)-(33) except that there are four copies of the gauge field fluctuations. Due to the chosen normalization of the $U(2)$ generators the gauge field background $\Phi$ enters with an additional factor $\frac{1}{2}$ compared to 30 . The quasinormal mode spectrum is the same as the one of the holographic s-wave superconductor [15] except that the scalar modes are doubly degenerate and the gauge field modes are fourfold degenerate. In particular there are four copies of the hydrodynamic diffusion mode $\omega=-i D k^{2}$.

\subsubsection{Perturbations in the Broken Phase}

The equations of motion in the broken phase decouple in two sets: one mixing the (0)-(3) colors of the gauge field and $\sigma$ fluctuations and the other mixing the $(1)-(2)$ colors and the $\eta$ fluctuations.

Writing $\sigma=\zeta+i \delta$, the equations of the $(0)-(3)$ sector are 


$$
\begin{array}{rl}
0= & f \zeta^{\prime \prime}+\left(f^{\prime}+\frac{2 f}{\rho}\right) \zeta^{\prime}+\left(\frac{\omega^{2}}{f}+\frac{\chi^{2}}{f}-\frac{k^{2}}{\rho^{2}}-m^{2}\right) \zeta+\frac{2 i \omega \chi}{f} \delta+\left(a_{t}^{(0)}-a_{t}^{(3)}\right) \Psi \frac{\chi}{f} \\
0= & f \delta^{\prime \prime}+\left(f^{\prime}+\frac{2 f}{\rho}\right) \delta^{\prime}+\left(\frac{\omega^{2}}{f}+\frac{\chi^{2}}{f}-\frac{k^{2}}{\rho^{2}}-m^{2}\right) \delta-\frac{2 i \omega \chi}{f} \zeta+i \Psi \omega \frac{a_{t}^{(3)}-a_{t}^{(0)}}{2 f}+ \\
& +i \Psi k \frac{a_{x}^{(3)}-a_{x}^{(0)}}{2 \rho^{2}} \\
0 & f a_{t}^{\prime \prime(0)}+\frac{2 f}{\rho} a_{t}^{\prime(0)}-\left(\Psi^{2}+\frac{k^{2}}{\rho^{2}}\right) a_{t}^{(0)}-\frac{\omega k}{\rho^{2}} a_{x}^{(0)}+\Psi^{2} a_{t}^{(3)}-4 \zeta \Psi \chi-2 i \omega \Psi \delta, \\
0 & f a_{x}^{\prime \prime(0)}+f^{\prime} a_{x}^{\prime(0)}+\left(\frac{\omega^{2}}{f}-\Psi^{2}\right) a_{x}^{(0)}+\frac{\omega k}{f} a_{t}^{(0)}+\Psi^{2} a_{x}^{(3)}+2 i k \delta \Psi, \\
0 & f a_{t}^{\prime \prime(3)}+\frac{2 f}{\rho} a_{t}^{\prime(3)}-\left(\Psi^{2}+\frac{k^{2}}{\rho^{2}}\right) a_{t}^{(3)}-\frac{\omega k}{\rho^{2}} a_{x}^{(3)}+\Psi^{2} a_{t}^{(0)}+4 \zeta \Psi \chi+2 i \omega \Psi \delta, \\
0= & f a_{x}^{\prime \prime(3)}+f^{\prime} a_{x}^{\prime(3)}+\left(\frac{\omega^{2}}{f}-\Psi^{2}\right) a_{x}^{(3)}+\frac{\omega k}{f} a_{t}^{(3)}+\Psi^{2} a_{x}^{(0)}-2 i k \delta \Psi, \\
0 & \frac{i k}{\rho^{2}} a_{x}^{\prime(0)}+\frac{i \omega}{f} a_{t}^{\prime(0)}+2 \Psi^{\prime} \delta-2 \Psi \delta^{\prime}, \\
0 & \frac{i k}{\rho^{2}} a_{x}^{\prime(3)}+\frac{i \omega}{f} a_{t}^{\prime(3)}-2 \Psi^{\prime} \delta+2 \Psi \delta^{\prime} .
\end{array}
$$

It is trivial to show that by defining new fields $a_{t}^{( \pm)} \equiv \frac{1}{2}\left(a_{t}^{(0)} \pm a_{t}^{(3)}\right)$ and $a_{x}^{( \pm)} \equiv \frac{1}{2}\left(a_{x}^{(0)} \pm\right.$ $\left.a_{x}^{(3)}\right)$ the system further decouples into a coupled system for the scalar fluctuations and $a_{\mu}^{(-)}$and a background independent set of equations for the $U(1)$ gauge field $a_{\mu}^{(+)}$. The first subsystem reproduces the eoms (37)-(41) and therefore corresponds to the s-wave $U(1)$ superconductor contained in the $U(2)$ model. On the other hand, the field $a_{\mu}^{(+)}$corresponds to the preserved gauge symmetry surviving the $U(2) \rightarrow U(1)$ spontaneous symmetry breaking. The quasinormal mode spectrum in this sector is therefore the same one as in [15] plus the QNMs that are stem from a $U(1)$ gauge field in $A d S_{4}$. In particular the hydrodynamic modes in this sector are the sound mode and the diffusion mode of the unbroken $U(1)$.

From now on we will concentrate on the remaining fields. We will call this remaining, inherently non-Abelian sector the $(1)-(2)$ sector and will show that the expected type II Goldstone boson resides there. Writing $\eta=\alpha+i \beta$, we find the following equations in the (1) - (2) sector: 


$$
\begin{array}{rl}
0= & f \alpha^{\prime \prime}+\left(f^{\prime}+\frac{2 f}{\rho}\right) \alpha^{\prime}+\left(\frac{\omega^{2}}{f}+\frac{(\Phi+\Theta)^{2}}{4 f}-\frac{k^{2}}{\rho^{2}}-m^{2}\right) \alpha+\frac{i \omega(\Phi+\Theta)}{f} \beta- \\
& -i \Psi\left(\frac{k}{2 \rho^{2}} a_{x}^{(2)}+\frac{\omega}{2 f} a_{t}^{(2)}\right)+\frac{\Psi \Phi}{2 f} a_{t}^{(1)}, \\
0= & f \beta^{\prime \prime}+\left(f^{\prime}+\frac{2 f}{\rho}\right) \beta^{\prime}+\left(\frac{\omega^{2}}{f}+\frac{(\Phi+\Theta)^{2}}{4 f}-\frac{k^{2}}{\rho^{2}}-m^{2}\right) \beta-\frac{i \omega(\Phi+\Theta)}{f} \alpha- \\
& -i \Psi\left(\frac{k}{2 \rho^{2}} a_{x}^{(1)}+\frac{\omega}{2 f} a_{t}^{(1)}\right)-\frac{\Phi \Psi}{2 f} a_{t}^{(2)}, \\
0= & f a_{t}^{\prime \prime(1)}+\frac{2 f}{\rho} a_{t}^{\prime(1)}-\left(\Psi^{2}+\frac{k^{2}}{\rho^{2}}\right) a_{t}^{(1)}-\frac{\omega k}{\rho^{2}} a_{x}^{(1)}+i \Theta \frac{k}{\rho^{2}} a_{x}^{(2)}-2 \Phi \Psi \alpha-2 i \omega \Psi \beta, \\
0 & f a_{x}^{\prime \prime(1)}+f^{\prime} a_{x}^{\prime(1)}+\left(\frac{\omega^{2}}{f}-\Psi^{2}+\frac{\Theta^{2}}{f}\right) a_{x}^{(1)}-2 i \frac{\Theta \omega}{f} a_{x}^{(2)}-i \Theta \frac{k}{f} a_{t}^{(2)}+\frac{\omega k}{f} a_{t}^{(1)} \beta+ \\
& +2 i k \Psi \beta, \\
0= & f a_{t}^{\prime \prime(2)}+\frac{2 f}{\rho} a_{t}^{\prime(2)}-\left(\Psi^{2}+\frac{k^{2}}{\rho^{2}}\right) a_{t}^{(2)}-\frac{\omega k}{\rho^{2}} a_{x}^{(2)}-i \Theta \frac{k}{\rho^{2}} a_{x}^{(1)}+2 \Phi \Psi \beta-2 i \omega \Psi \alpha, \\
0= & f a_{x}^{\prime \prime(2)}+f^{\prime} a_{x}^{\prime(2)}+\left(\frac{\omega^{2}}{f}-\Psi^{2}+\frac{\Theta^{2}}{f}\right) a_{x}^{(2)}+2 i \frac{\Theta \omega}{f} a_{x}^{(1)}+i \Theta \frac{k}{f} a_{t}^{(1)}+\frac{\omega k}{f} a_{t}^{(2)}+ \\
& +2 i k \Psi \alpha, \\
0= & \frac{i k}{\rho^{2}} a_{x}^{\prime(1)}+\frac{i \omega}{f} a_{t}^{\prime(1)}+\frac{1}{f}\left(a_{t}^{\prime(2)} \Theta-a_{t}^{(2)} \Theta^{\prime}\right)+2 \Psi^{\prime} \beta-2 \beta^{\prime} \Psi \\
0 & \frac{i k}{\rho^{2}} a_{x}^{(2)}+\frac{i \omega}{f} a_{t}^{\prime(2)}-\frac{1}{f}\left(a_{t}^{\prime(1)} \Theta-a_{t}^{(1)} \Theta^{\prime}\right)+2 \Psi^{\prime} \alpha-2 \alpha^{\prime} \Psi .
\end{array}
$$

A comment is in order here. This system of equations could be written in a more compact form by using complex field variables $\eta$ and $a_{t, x}^{(1)} \pm i a_{t, x}^{(2)}$. One has to keep in mind then that the field equations one needs to solve for the QNM spectrum for the complex conjugate fields are not the complex conjugate equations since one has to demand infalling boundary conditions on the fields and on the complex conjugate fields simultaneously. This aspect is somewhat clearer if one works with the (formally) real field variables on paying the price of writing a somewhat lengthy system of equations.

Up to linear order in perturbations, there are three decoupled sectors in the system. Two of them belong to the '(0) - (3) sector' and they are a copy of the $U(1)$ holographic superconductor, already extensively studied, and the preserved $U(1)$ gauge symmetry. The main features of the spectrum of this sector have already been presented in section 3 since it is also a subsector of the ungauged model. On the other hand, the so called '(1) - (2) sector' has not been studied before. The physics in this sector is quite different from the holographic superconductors studied up to now and we will concentrate on it in the rest of this paper. 
Before studying the quasinormal modes we will focus on a simpler problem, namely the conductivities.

\subsection{Conductivities}

In order to study the conductivities via Kubo formulae, it is enough to solve the linearized equations in the limit $k=0$. The retarded correlators that we are interested in have the form $\mathcal{G}_{\mathcal{R}} \sim\left\langle J_{(c)}^{x}, J_{\left(c^{\prime}\right)}^{x}\right\rangle_{\mathcal{R}}$, with $c, c^{\prime}$ color indices.

We will be applying the prescription of [34] for computing Green functions in the presence of holographic operator mixing. If one has a set of fields $\Phi_{I}$, the two-point correlation functions will be

$$
\mathcal{G}_{I J}=\lim _{\Lambda \rightarrow \infty}\left(\mathcal{A}_{I M} \mathcal{F}_{k}^{M}{ }_{J}(\Lambda)^{\prime}+\mathcal{B}_{I J}\right)
$$

where the matrix $\mathcal{F}_{k}(r)$ is nothing but the bulk-to-boundary propagator for the fields, normalized to be the unit matrix at the boundary. The matrices $\mathcal{A}$ and $\mathcal{B}$ can be read off from the on-shell renormalized action. The corresponding DC conductivities are given by the following Kubo formula

$$
\sigma_{I J}=\lim _{\omega \rightarrow 0}\left(\frac{i}{\omega} \mathcal{G}_{I J}(\omega, 0)\right) .
$$

At vanishing momentum the longitudinal components of the gauge field perturbations decouple from the scalar perturbations, as well as from the temporal components of the gauge fields. Moreover, the constraints (eqs. (81, 82) and (89, 90) become trivial. Since we know that the system splits into the $(0)-(3)$ and the $(1)-(2)$ sectors we can rearrange the $a_{x}^{(c)}$ fields in two vectors

$$
\Phi_{k(0-3)}^{T}(\rho)=\left(a_{x}^{(0)}(\rho), a_{x}^{(3)}(\rho)\right) \quad \text { and } \quad \Phi_{k(1-2)}^{T}(\rho)=\left(a_{x}^{(1)}(\rho), a_{x}^{(2)}(\rho)\right) .
$$

One can check that in our case the $\mathcal{A}, \mathcal{B}$ matrices take the simple form

$$
\mathcal{A}=-\frac{f(r)}{2} \mathbb{I}, \quad \mathcal{B}=0
$$

for both sectors. A priori we would have a $4 \times 4$ matrix of conductivities. We know however that the fluctuations in the $(0)-(3)$ and the $(1)-(2)$ sector decouple from each other. Therefore we can restrict ourselves to study two independent $2 \times 2$ matrices of conductivities.

\subsection{Conductivities in the $(0)-(3)$ sector}

The $k=0$ equations of motion for $a_{x}^{(0)}$ and $a_{x}^{(3)}$ can be simplified by using the already defined $a_{x}^{(-)}$and $a_{x}^{(+)}$fields. This results in

$$
\begin{aligned}
& 0=f a_{x}^{\prime \prime(+)}+f^{\prime} a_{x}^{(+)}+\frac{\omega^{2}}{f} a_{x}^{(+)} \\
& 0=f a_{x}^{\prime \prime(-)}+f^{\prime} a_{x}^{\prime(-)}+\left(\frac{\omega^{2}}{f}-2 \Psi^{2}\right) a_{x}^{(-)} .
\end{aligned}
$$


We see that the resulting system of equations is now completely decoupled. We only have two diagonal conductivities $\sigma_{++}$and $\sigma_{--}$, corresponding to the unbroken $U(1)$ diffusive sector and a mode which is associated to the broken $U(1)$ coupling to the condensate. The former is the same as in the unbroken phase and of no further interest for us. The latter is again the well-studied $U(1)$ s-wave superconductor. Its conductivity has been already calculated in [3]. To check our numerics we have re-calculated it and in Figure 11 we show its behavior. It coincides completely with [3]. The real part shows the $\omega=0$ delta function characteristic of superconductivity ${ }^{8}$. Numerically this can be seen through the $1 / \omega$ behavior in the imaginary part. The Kramers-Kronig relation (see (121) in appendix A) implies then infinite DC conductivity. The real part of the AC conductivity also exhibits a temperature dependent gap.
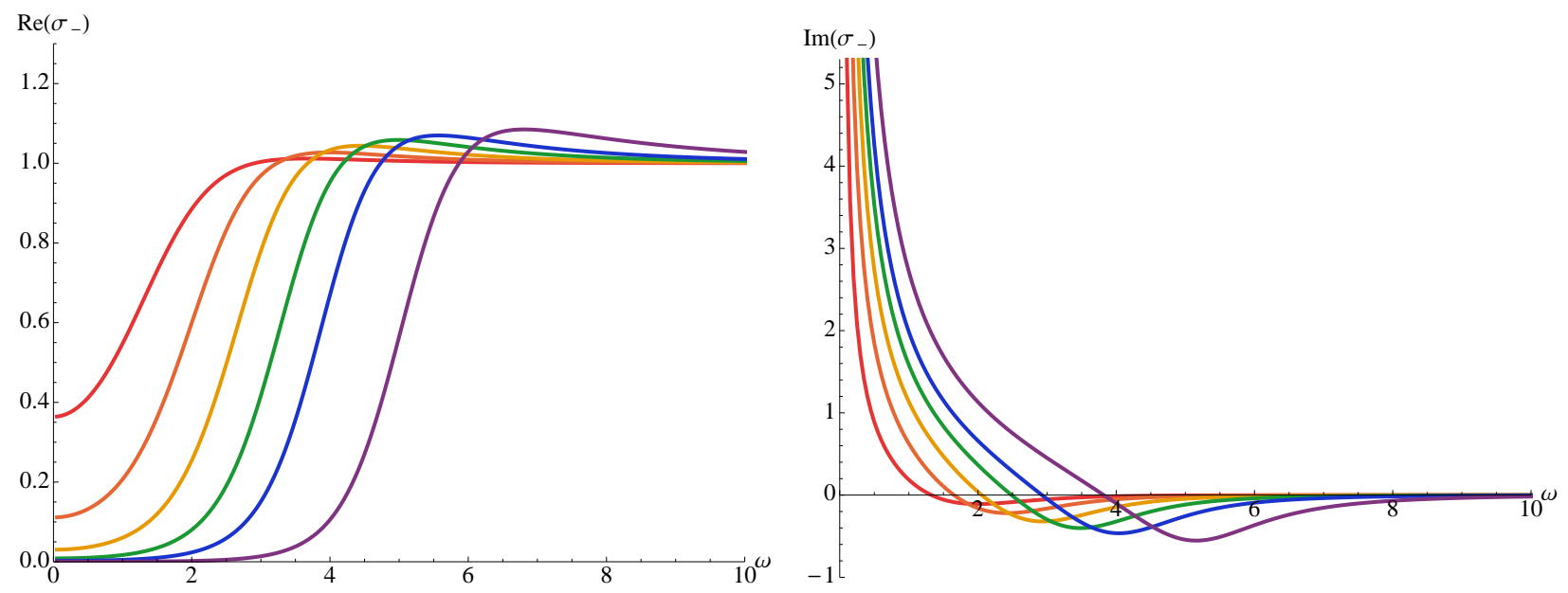

Figure 11: Real part (left) and imaginary part (right) of the conductivity as a function of frequency. The plots correspond to temperatures in the range $T / T_{c} \approx 0.91-0.41$, from red to purple. As expected, the plots reproduce the ones of [3].

\subsection{Conductivities in the $(1)-(2)$ sector}

The relevant equations for the $(1)-(2)$ sector read

$$
\begin{aligned}
& 0=f a_{x}^{\prime \prime(1)}+f^{\prime} a_{x}^{\prime(1)}+\left(\frac{\omega^{2}}{f}-\Psi^{2}+\frac{\Theta^{2}}{f}\right) a_{x}^{(1)}-2 i \frac{\Theta \omega}{f} a_{x}^{(2)} \\
& 0=f a_{x}^{\prime \prime(2)}+f^{\prime} a_{x}^{(2)}+\left(\frac{\omega^{2}}{f}-\Psi^{2}+\frac{\Theta^{2}}{f}\right) a_{x}^{(2)}+2 i \frac{\Theta \omega}{f} a_{x}^{(1)} .
\end{aligned}
$$

These equations obey the symmetry

$$
\left(a_{x}^{(1)} \rightarrow a_{x}^{(2)}, a_{x}^{(2)} \rightarrow-a_{x}^{(1)}\right) .
$$

\footnotetext{
${ }^{8}$ In general, this behavior is also typical of translation invariant charged media, in which accelerated charges cannot relax. However, working in the probe limit we effectively break translation invariance and therefore the infinite DC conductivity is a genuine sign of superconductivity.
} 
One can see that the fact that $\Theta(1)=0$ implies that $a_{x}^{(1)}(1)$ is independent of $a_{x}^{(2)}(1)$, so, after imposing infalling boundary conditions at the horizon, the parameter space of boundary conditions is two-dimensional, as expected.

In the unbroken phase the system completely decouples

$$
0=f a_{x}^{\prime \prime(c)}+f^{\prime} a_{x}^{\prime(c)}+\frac{\omega^{2}}{f} a_{x}^{(c)}
$$

\subsubsection{Diagonal Conductivities $\sigma^{11} \& \sigma^{22}$}

The diagonal components of the conductivity, $\sigma^{11}$ and $\sigma^{22}$ have the same behavior, as could be anticipated from the equations $(97),(98)$. Henceforth, we will only refer to $\sigma^{11}$, but all the conclusions also apply to $\sigma^{22}$.

Figure 12 shows the conductivity for several values of the temperature. We find that these conductivities also show delta-function singularities at $\omega=0$.
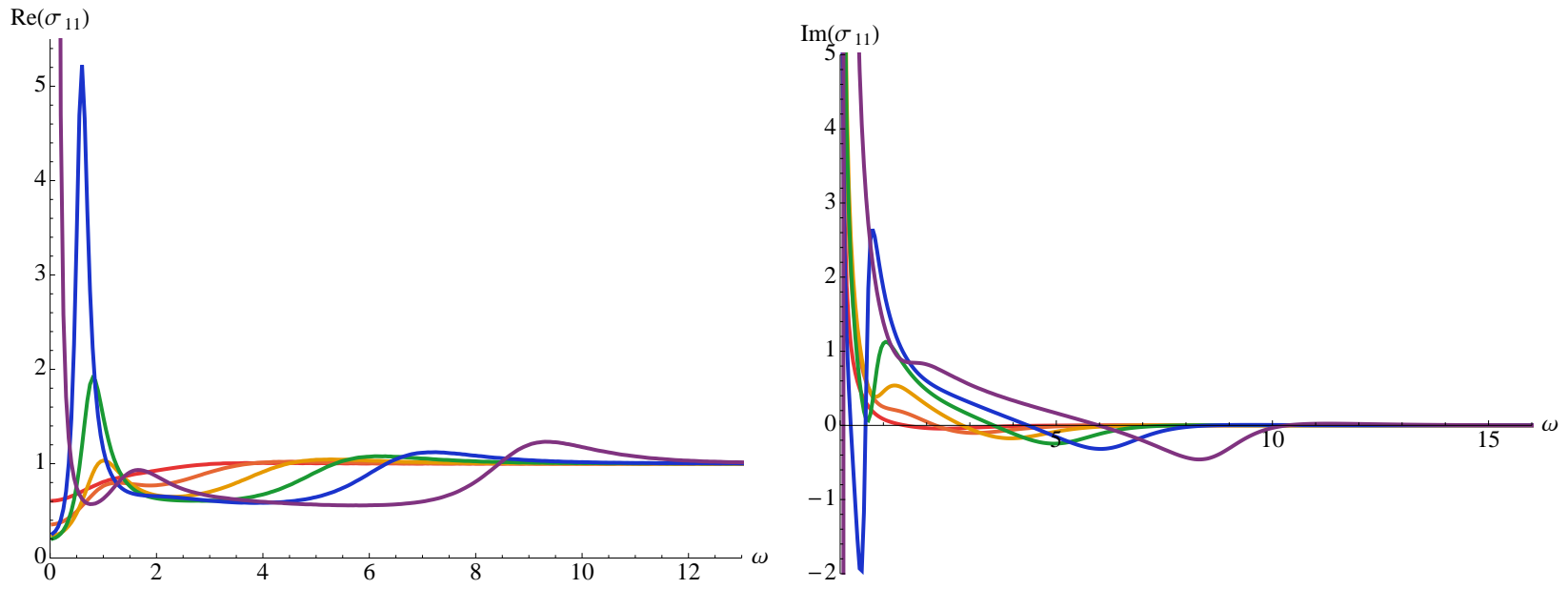

Figure 12: Real (left) and imaginary (right) parts of $\sigma^{11}$ versus $\omega$ for five different temperatures chosen in a range $T / T_{c} \approx 0.91-0.41$, from red to purple. $\operatorname{Im}\left(\sigma^{11}\right)$ clearly blows up as $\omega \rightarrow 0$.

The strength of the delta function can also be computed. It is given by the residue of the imaginary part of the conductivity at $\omega=0$,

$$
\lim _{\omega \rightarrow 0} \omega \operatorname{Im}\left(\sigma^{11}\right) \sim n_{s}
$$

The residue is plotted in Figure 13 as a function of $T / T_{c}$. As expected, it starts growing from a zero value. At $T / T_{c} \approx 0.65, n_{s}$ reaches a maximum and starts decreasing fast, changing sign at $T / T_{c}=0.49$. To study $n_{s}$ down to very low temperature we would need to go beyond the proble limit. However, as we will comment below, this behaviour of $n_{s}$ can be understood in light of the QNM spectra. 


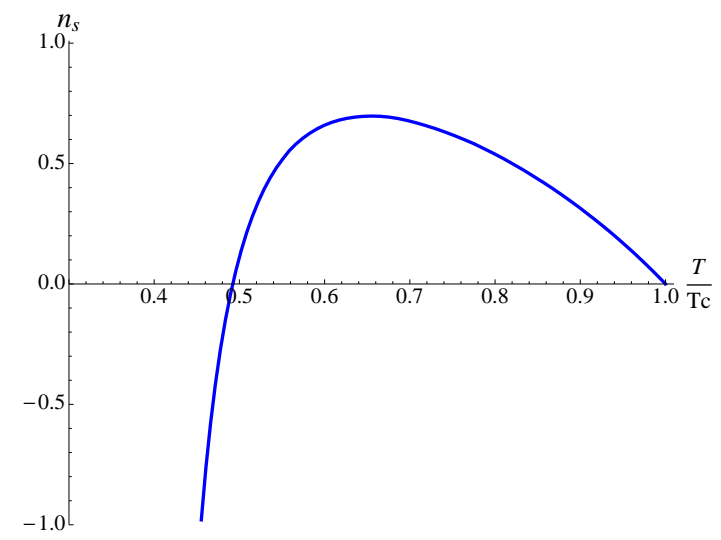

Figure 13: Residue at $\omega=0$ as a function of $T / T_{c}$.

Let us look in detail at the behavior of the real part of the conductivity (left plot in figure 12.). For high enough temperatures the optical conductivity is almost constant, $\operatorname{Re}\left(\sigma^{11}\right)=1$, which is expected since in that regime the dynamics is described essentially by $(100)$. As soon as we decrease the temperature, the onset of the DC conductivity also decreases and only approaches the constant value asymptotically, when $\omega$ becomes large enough and thus the term $\frac{\omega^{2}}{f}$ dominates, turning equations $(97), 988$ approximately into (100). According to the Ferrell-Glover sum rule, the area missing as we lower the temperature is proportional to $n_{s}$.

Interestingly, at low temperatures the real part of $\sigma^{11}$ starts developing a bump at small values of $\omega(0<\omega \lesssim 2)$. The bump leaves less area for the delta function to cover, which explains why $n_{s}$ starts decreasing approximately at this temperature. Moreover, the appearance of these bumps can be traced back to the fact that for a subleading gauge QNM with small $|\operatorname{Im}(\omega)|, \operatorname{Re} \omega(T)>>\operatorname{Im} \omega(T)$ holds. Hence, the conductivities affected by this mode start developing the reminiscence of a resonance at a particular frequency. We have studied the spectrum of low lying QNM for the gauge sector and found that this mode corresponds in the normal phase to the lowest excitation of $a_{\mu}^{(1,2)}, \omega=-1.5 i$. But it is at lower temperatures where one finds a remarkable fact: at $T / T_{c} \approx 0.395$ the mode becomes unstable, and indeed, as we will see, several physical quantities modify their behavior at that temperature.

Therefore, we expect a new phase transition around $T / T_{c} \approx 0.395$, due entirely to the (1) - (2) sector. Since this phase transition seems to be triggered by an unstable mode in the vector sector it most likely leads to the formation of a p-wave condensate. This surely requires further investigation that is currently underway [55].

\subsubsection{Off-diagonal conductivities $\sigma^{12} \& \sigma^{21}$}

The off-diagonal elements of the conductivity matrix are also related via the symmetry 99) and therefore obey $\sigma^{12}=-\sigma^{21}$. Therefore, it is enough to comment on $\sigma^{12}$, although the conclusions are valid for both components.

The form of $\sigma^{12}$ is plotted in Figure 14 for various different temperatures as a function 
of frequency. At $T / T_{c}=1$ the system is practically decoupled, so for all temperatures the off-diagonal conductivity goes to zero as $\omega$ increases.
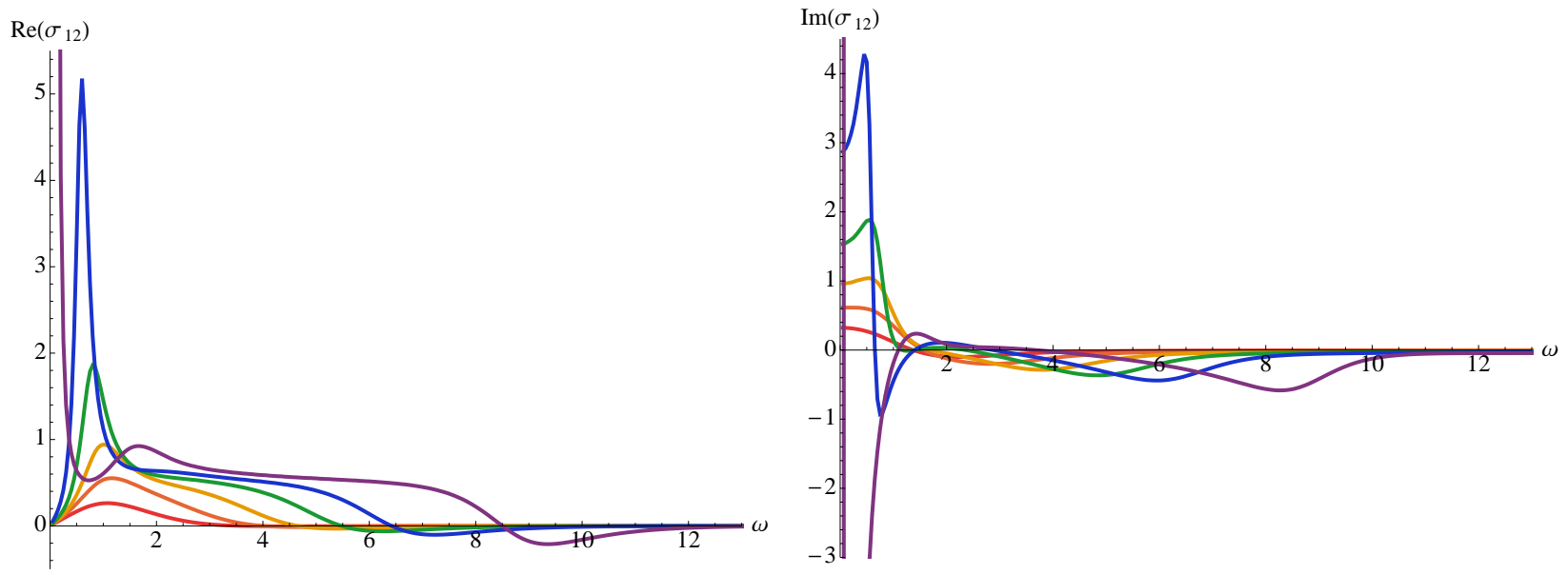

Figure 14: Real (left) and imaginary (right) part of $\sigma^{12}$ as a function of $\omega$ for $T / T_{c} \approx$ $0.91-0.41$, from red to purple.

Observe that $\sigma^{12}(\omega)$ behaves as a normal conductivity. Its real part vanishes as $\omega \rightarrow 0$, whereas the imaginary part tends to a constant value.

\subsubsection{Conductivities $\sigma_{+-}$and $\sigma_{-+}$}

It is worth to notice that the equations (97)-(98) decouple if we define a new vector field

$$
\tilde{\varphi}=\left(\begin{array}{c}
A_{+} \\
A_{-}
\end{array}\right)=\left(\begin{array}{cc}
1 & i \\
1 & -i
\end{array}\right)\left(\begin{array}{l}
a_{x}^{(1)} \\
a_{x}^{(2)}
\end{array}\right)=S \varphi .
$$

In this basis, the equations of motion become

$$
0=f A_{ \pm}^{\prime \prime}+f^{\prime} A_{ \pm}^{\prime}+\left(\frac{(\omega \mp \Theta)^{2}}{f}-\Psi^{2}\right) A_{ \pm} .
$$

It is easy to check that the relation between the conductivity matrices in the two basis is given by

$$
\tilde{\sigma}=\left(S^{T}\right)^{-1} \sigma S^{-1}
$$

and that only the off-diagonal components of $\tilde{\sigma}$ are non vanishing.

The conductivities $\sigma_{-+}$and $\sigma_{+-}$are represented in Figure 15 and 16 , respectively. The plot of the conductivity $\sigma_{-+}$is particularly suggestive. Besides the superconducting delta of the DC conductivity, it resembles the behavior observed in Graphene [29]. Such a resemblance of the conductivities of holographic superconductors to the one of graphene has been pointed our already in [37]. We emphasize however that the conductivities shown in figure 15 have an even closer resemblance to [29]. In particular, at small frequencies we see that 
a Drude-like peak develops. This kind of behavior in metals is usually due to the presence of impurities or lattices, whereas in our case, momentum relaxation would be due to the non-vanishing expectation value of the charge density operator $\left\langle Q_{3}\right\rangle=\bar{n}_{\Theta}$. The resemblance holds for not too low temperatures. When lowering the temperature, a gap opens up as for the $(0)-(3)$ sector. The real part of $\sigma_{+-}$shows the same peak already observed for $\sigma_{11}$ when decreasing the temperature. For temperatures below $T \approx 0.49 T_{c}$, the pole in the imaginary part of both conductivities changes sign. Of course, it corresponds to the temperature at which the residue changes sign. The onset of the DC conductivity at low temperatures grows very fast, becoming divergent at $T / T_{c} \approx 0.395$. The presence of such a pole in the conductivity is related to the appearance of an instability in the spectrum of excitations of the gauge field and therefore with a phase transition to another superconducting phase, as already discussed.
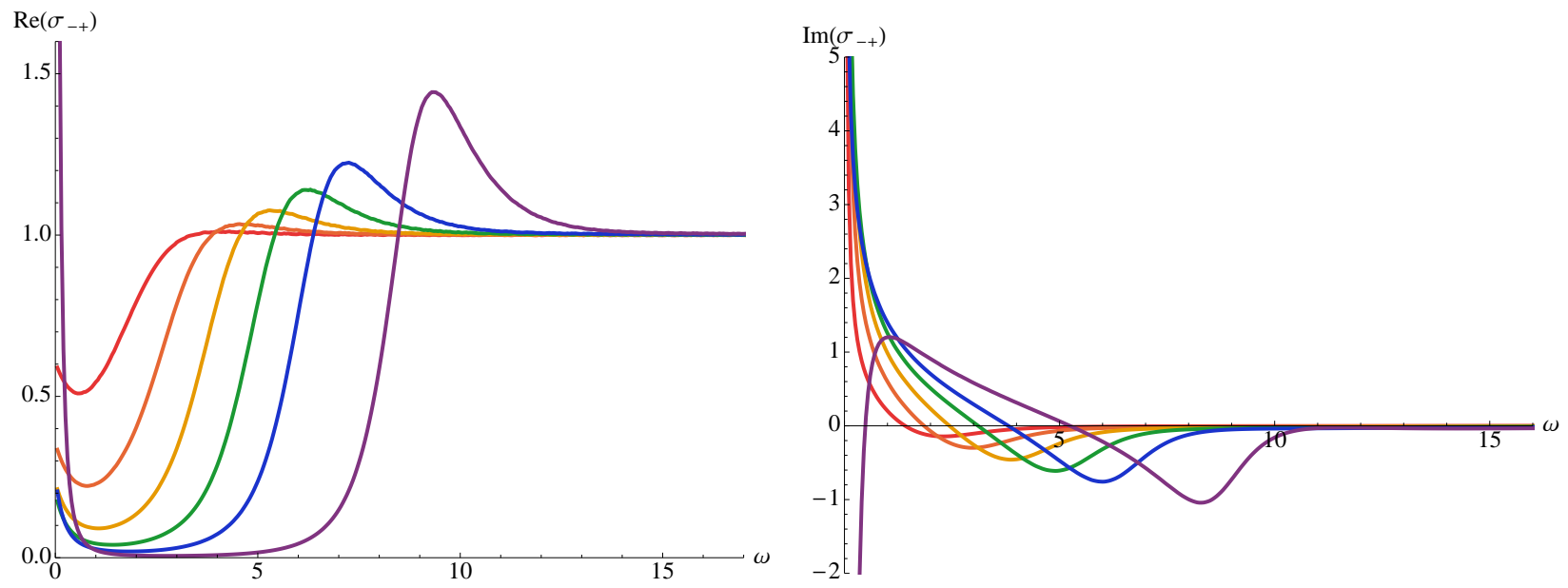

Figure 15: Real (left) and imaginary (right) part of the conductivity $\sigma_{-+}$for temperatures in the range $T / T_{c} \approx 0.91-0.41$, from red to purple.

\subsection{Quasinormal Modes}

Let us finally study the QNM spectrum in the (1) - (2) sector. This sector contains the fluctuations $\eta, a_{\mu}^{i}$ with $i=1,2$, therefore in the unbroken phase the spectrum will contain two diffusive modes associated with the two gauge fields. The fluctuations of the scalar field in the normal phase were already discussed in section 3. Analyzing the quasinormal mode spectrum in the broken phase amounts to solving the system of equations (83)-(90). Details of the computation can be found in appendix B. 

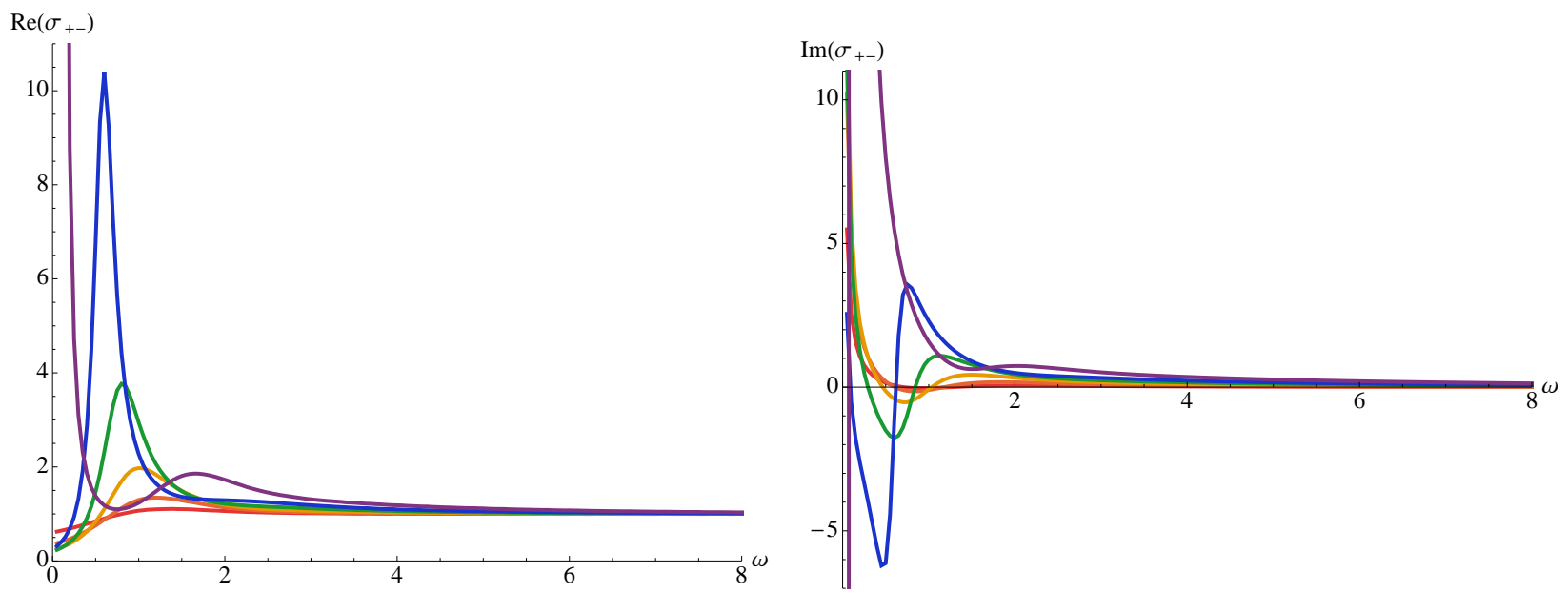

Figure 16: Real (left) and imaginary (right) part of the conductivity $\sigma_{+-}$for temperatures in the range $T / T_{c} \approx 0.91-0.41$, from red to purple.

\subsubsection{Type II Goldstone mode}

As expected within the (1) - (2) sector we find a type II Goldstone mode. As in the ungauged model for small enough momentum its dispersion relation can be fitted to

$$
\omega= \pm \mathcal{B} k^{2}-i \mathcal{C} k^{2} .
$$

Figure 17 shows the dispersion relation for various values of the temperature in the hydrodynamic regime. The quadratic behavior with momentum is apparent.
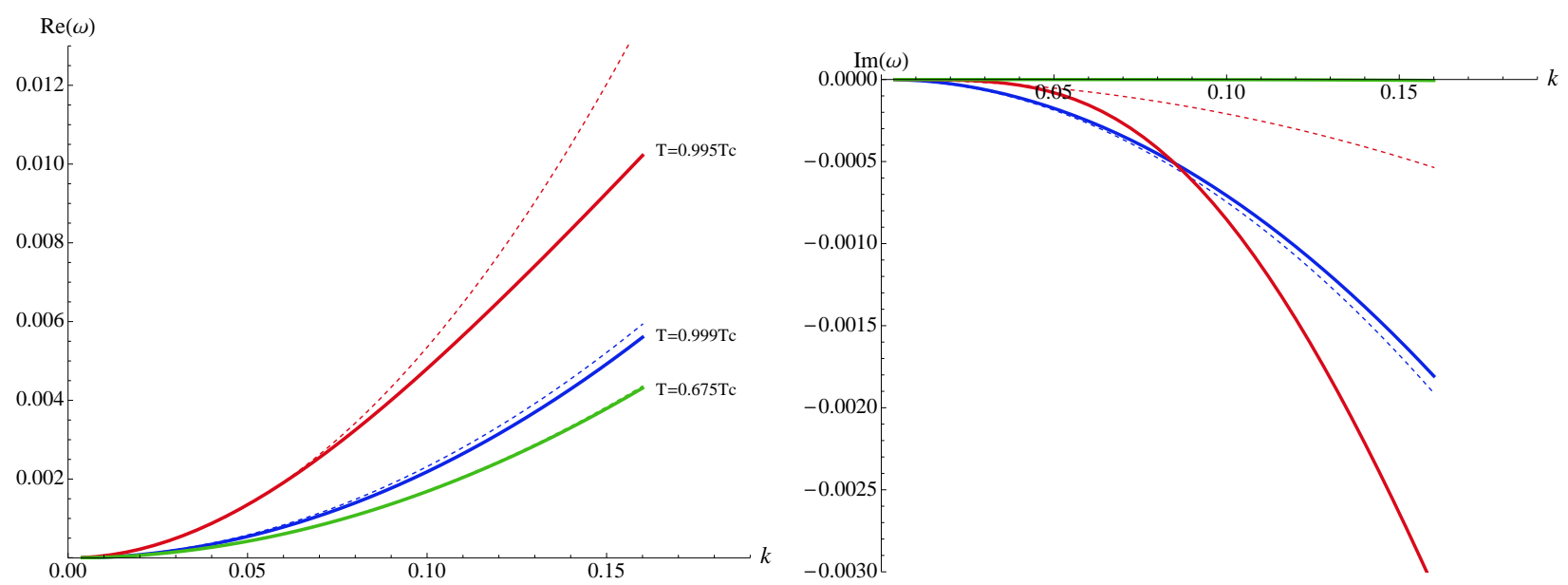

Figure 17: Plots of $\operatorname{Re}(\omega)$ (left) and $\operatorname{Im}(\omega)$ (right) as a function of the momentum. Thick lines correspond to data and thin lines to quadratic fit. At $T=0.995 T_{c}$ the real quadratic parameter $\mathcal{B}(T)$ shows a maximum, see Figure 18. Relation (105) is fulfilled with high accuracy. 
The temperature dependence of $\mathcal{B}$ and $\mathcal{C}$ is plotted in Figure 18 . The value at $T=T_{c}$ is given by the same value as in the ungauged model (46) and in fact can also be cross checked by calculating the scalar mode dispersion relation in the unbroken phase at $T=T_{c}$ since the QNMs must be continuous through the phase transition. We find a rather surprising dependence of $\mathcal{B}$ with the temperature. It starts at a finite value at the transition and then it rises rather sharply and falls off slower. It reaches a minimum at $T \approx 0.49 T_{c}$, temperature at which we found the change of sign in the residue of current-current correlators. We also find another peak around $T \approx 0.4 T_{c}$. We expect that it is again related with the instability found in the gauge sector around that temperature. It would also be interesting to calculate $\mathcal{B}(T)$ using an alternative method e.g. as the sound velocity can be calculated from thermodynamic considerations alone. In order to do this one would need to formulate the hydrodynamics of type II Goldstone modes. We are however not aware of such a hydrodynamic formulation and leave this for future research.
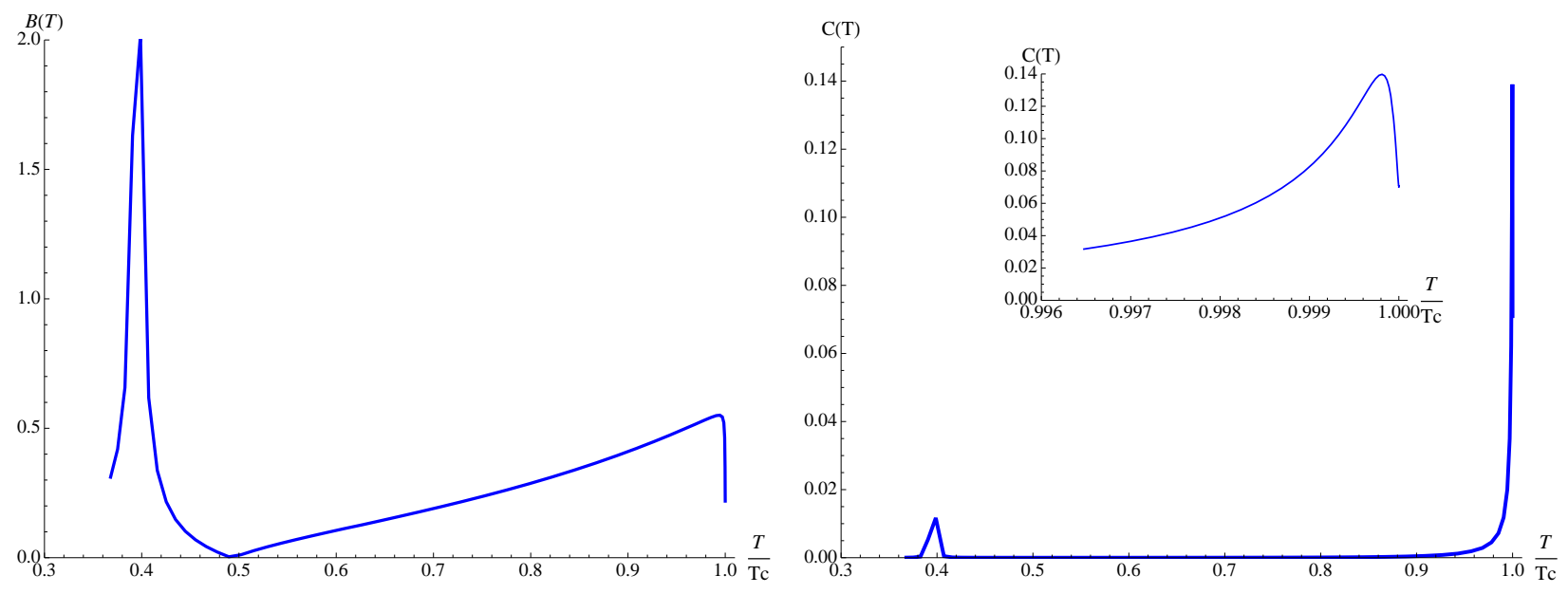

Figure 18: $\mathcal{B}$ (left) and $\mathcal{C}$ (right) as a function of $T / T_{c}$. The zoom-in shows the peak of $\mathcal{C}$ close to the transition. Furthermore at $T \simeq 0.4 T_{c}$ a sharp peak shows up in both coefficients. We relate this feature also to the instability arising in the vector sector.

The attenuation $\mathcal{C}(T)$ decreases rapidly with temperature. For temperatures $T / T_{c}<0.9$ it is negligible and the width of the type II Goldstone scales with $k^{4}$ in the hydrodynamic limit. This fast decreasing with temperature reflects that this mode propagates almost ideally in the fluid at low temperature. No further ungapped modes can be found in this sector.

\subsubsection{Higher quasinormal modes}

Higher quasinormal modes correspond to gapped modes in the QNM spectrum and thus represent subleading contributions to the low energy Green's functions. We will focus here only on two of them: the continuation of the two diffusive modes of the unbroken phase and the special gapped mode that appears as the partner mode of the type II Goldstone mode in the field theoretical model. 
Analyzing the first one is interesting in order to understand if also a qualitative change in the response pattern, such as that characterized by $T_{*}$ in the $U(1)$ superconductor sector, exists in the (1) - (2) sector. Since in this sector there exist however two diffusive modes in the unbroken phase it is also possible that the diffusive modes do not simply develop a gap but that they pair up and move off the imaginary axis in the broken phase. Indeed as we will see this is what happens.

The special gapped mode corresponds to a mode that is associated to the complex conjugate of the scalar perturbation in the unbroken phase. At $k=0$ and $\mu=0$ the scalar mode and its complex conjugate are degenerated. As we lower the temperature they split into two different modes. When we reach $T=T_{c}$, the lowest scalar mode becomes the type II Goldstone mode whereas the mode of the complex conjugate scalar field turns into the special gapped mode. The gap of this mode is expected to be given by the tree level result (13) [31.

Fate of diffusive modes: As already mentioned, in the (1) - (2) sector we have two degenerate diffusive modes in the unbroken phase. When going through the phase transition these modes can therefore pair up and move off the imaginary axes such that their quasinormal frequencies develop real parts and lie symmetrically around the imaginary axis. We expect therefore that in the low energy limit the dispersion relation takes the form

$$
\omega=\Gamma(T)+\mathcal{M}(T) k^{2},
$$

where both coefficients are complex functions and the second mode is located at $\omega^{\prime}=-\omega^{*}$. Besides, we expect the QNMs to be continuous through the phase transition, which in particular means that for $T=T_{c}$, our pseudo-diffusive modes should match the unbroken phase values, i.e. $\Gamma\left(T_{c}\right)=0$ and $\mathcal{M}\left(T_{c}\right)=-i$.

The modes at zero momentum are plotted in Figure 19. We see that indeed the gap vanishes as $T \rightarrow T_{c}$, whereas the modes split and develop a real part as we decrease the temperature. This last feature is exclusive of the non-Abelian system and thus does not take place in the usual $U(1)$ holographic superconductor, where the gap is purely imaginary (see [15] and comments above). Close to the phase transition, they present a linear behavior in temperature,

$$
\Gamma(T)=(4.1-0.8 i)\left(1-\frac{T}{T_{c}}\right) \quad \text { near } T_{c} .
$$

The temperature dependence of the coefficient of the momentum in $(106), \mathcal{M}(T)$, is shown in Figure 20. The real part rises very steeply just below the phase transition. The imaginary part approaches the unbroken phase value at the critical temperature, i.e. $\mathcal{M}\left(T_{c}\right)=-i$, as is expected for the pseudo-diffusion modes to continuously connect to the normal diffusion modes through the phase transition. Notice $\operatorname{Im} \mathcal{M}(T)$ decreases when lowering the temperature.

Another check of the fact that the pseudo diffusion modes come from the pairing up of the diffusion modes of the normal phase is that their dispersion relation at the phase transition matches. Therefore the two diffusive modes are continuous through the transition, as 


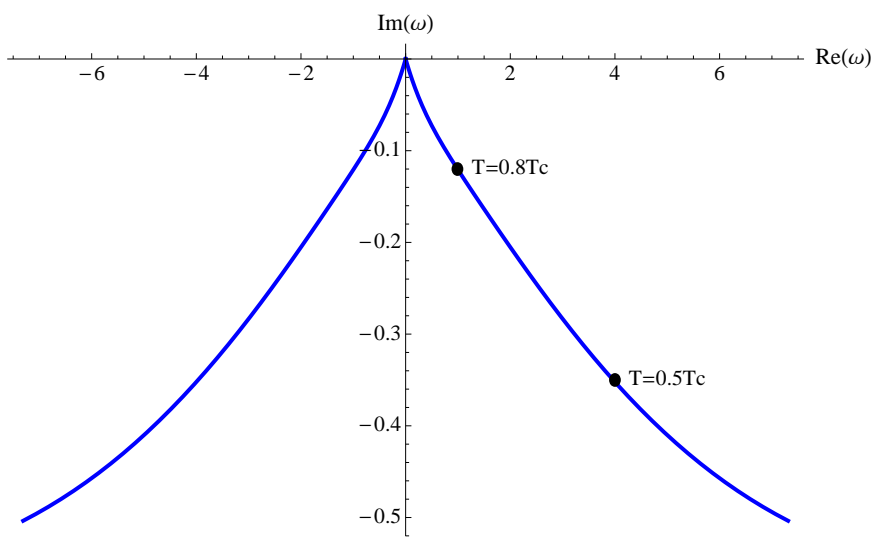

Figure 19: $\operatorname{Im} \omega$ versus $\operatorname{Re} \omega$ at $k=0$ as a function of the temperature. The shape of the figure is compatible with $T$ symmetry, since there are two pseudo-diffusive modes. Having $\operatorname{Re} \omega(k=0) \neq 0$ is characteristic of the non-Abelian case.
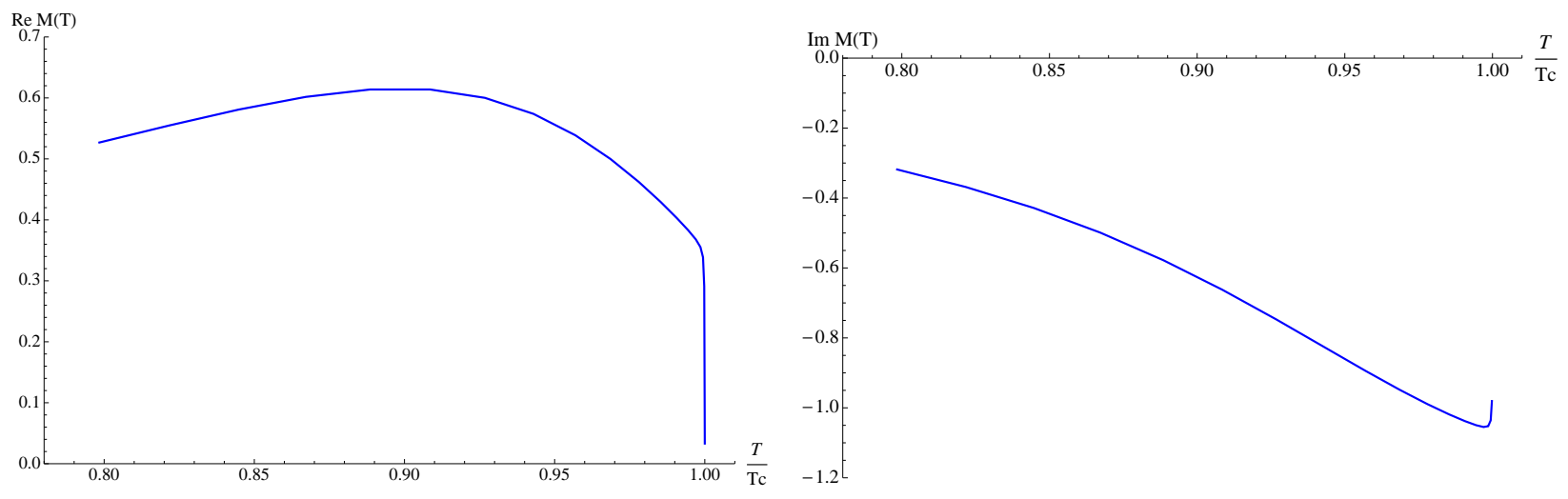

Figure 20: Real (left) and imaginary (right) part of $\mathcal{M}(T)$ as a function of $T / T_{c}$. As the temperature approaches $T_{c}$, the value of $\mathcal{M}(T)$ reaches the one prescribed by continuity through the phase transition. 
expected for second order phase transitions, however instead of simply developing an imaginary gap to drop out of the hydrodynamic spectrum as for the usual $U(1)$ superconductor, they pair up in two modes that on top of this gap also develop a real part.

The fact that $\operatorname{Re}(\omega)$ does not vanish for these modes implies that sufficiently close to $T_{c}$ and in the limit $k=0$, the late-time response of the perturbed state will present an oscillatory decay of the perturbations, meaning that, contrary to the $U(1)$ case, there will not be a temperature at which the late-time behavior changes qualitatively.

Special Gapped mode: Seeking for this mode is computationally much more involved. Its behavior is characterized by a gap that is proportional to $\mu$. In particular, in [31] it was argued that a type II Goldstone mode is accompanied by a gapped mode obeying $\omega(0)=q \mu$ with $q$ being the charge of the corresponding field. In our conventions here we have $q=1$. So we have to look for a mode with $\omega(k=0)=\mu$. Furthermore we expect that it connects to the lowest mode of the complex conjugate scalar in the unbroken phase.

In Figure 21 we depict such mode at zero momentum with respect to the chemical potential $\bar{\mu}$ in numerical units. Notice that the mode is continuous at the phase transition, as expected. We observe the linear behavior with the chemical potential that is predicted theoretically, at least near $\bar{\mu}_{c}$. It is very difficult to do the analysis when $\bar{\mu}>6$ due to the high computational power demanded to carry out the computation. The mode shows of course also a non-vanishing imaginary part which is due to the dissipation at finite temperature. We find that the real part above the phase transition can be approximated by

$$
\operatorname{Re} \omega=1.10 \bar{\mu} \text { near } \bar{\mu}_{c} \text {. }
$$

This result shows a deviation from the conjectured behavior which could nevertheless be due to uncertainties in the numerics. Let us emphasize here that the numerics involved in tracking this mode through the phase transition were rather challenging.
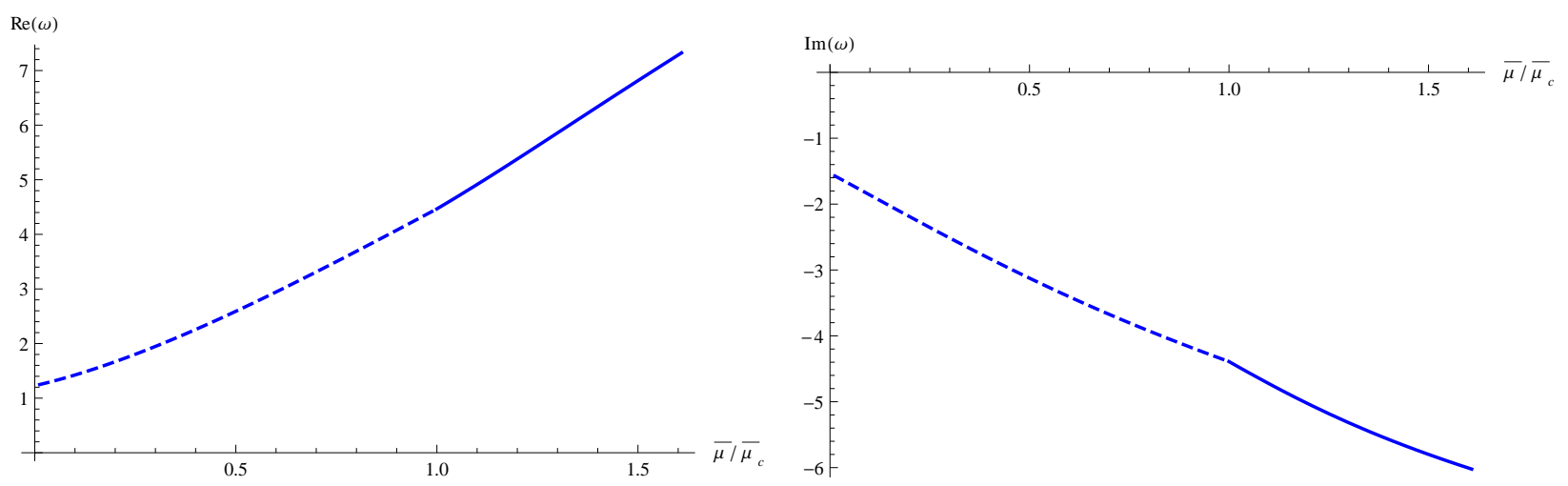

Figure 21: Real (left) and imaginary (right) part of the special gapped mode versus the chemical potential. We encounter the expected linear behavior with $\mu$. The plot covers both the unbroken (dashed line) and the broken (solid line) phases. 


\section{$5 \quad$ Discussion and Outlook}

The main focus of this work was to establish the existence of type II Goldstone modes in the quasinormal mode spectrum of a holographic theory dual to a strongly coupled superfluid with $U(2)$ symmetry.

We studied two models, one in which only the overall $U(1)$ symmetry is gauged in the AdS bulk and another in which all the $U(2)$ symmetry is gauged. The most important finding is that indeed there exist ungapped excitations represented by quasinormal modes in the AdS bulk that show the expected but somewhat unusual quadratic dispersion relation of type II Goldstone bosons.

For the ungauged model this does constitute a surprising result. After all, the field theory dual to this model does not contain the necessary conserved currents that would correspond to the generators of the global $S U(2)$ symmetry. Standard proofs of the Goldstone theorem take the existence of such conserved currents for granted. On the other hand it is basically guaranteed that one can construct an effective field theory, a simple LandauGinzburg type model, that captures the essential dynamics of the light modes, i.e. the lowest lying quasinormal modes. Such a model would be essentially given by the field theoretical model of section 2 and this guarantees the existence of the type II Goldstone modes. However one can expect that such an effective field theory approach can capture only the physics of the low lying QNMs but not the higher modes. This is indeed what happens: the partner mode of the type II Goldstone mode in the ungauged model does not behave in the supposed universal way $\omega=q \mu$. In contrast the corresponding mode in the gauged model does obey this relation approximately and the deviation we found could very well be attributed to numerical difficulties and uncertainties that arise in the study of the higher QNMs.

One rather interesting perspective on the ungauged model opens up if we vary the masses of the scalar fields in the AdS bulk. If the masses are slightly different, then at the critical temperature only one of the two scalars will feature an ungapped QNM (the one with smaller mass). The lowest scalar mode of the second one will still be gapped at that temperature. As one goes through the phase transition we do not expect this mode to become massless at lower temperatures. Rather it should become a pseudo-Goldstone mode with a gap that is proportional to the mass splitting. The appearance of the type II Goldstone mode can then be interpreted as the effect of a symmetry enhancement at the point in parameter space where the masses of the scalars become degenerate. Since this symmetry is not represented by bulk-gauge fields we might call it an accidental symmetry. At this point it is difficult to resist the temptation to draw a parallel to the conjectured symmetry enhancement of high $T_{c}$ superconductors. In [38] it was suggested that the phase diagram of high $T_{c}$ superconductors can be captured by a unified model with and enhancement of the $S O(3) \times U(1)$ symmetry of rotations and electromagnetism to a larger $S O(5)$ symmetry. Since high $T_{c}$ superconductors are d-wave rather than s-wave it remains to be seen how our symmetry enhancement mechanism and the resulting type II Goldstone mode can be combined with holographic models of d-wave superfluids such as [5, 6] 9 .

\footnotetext{
${ }^{9}$ The appearance of unexpected massless modes related to symmetry enhancement in the context of Bose
} 
The second model we studied has bulk gauge fields for all of the $U(2)$ symmetry. There are several important differences compared to the ungauged model. The most eye-jumping one is that now we can also define and study the full set of conductivities corresponding to the $U(2)$ symmetry. Nothing special occurs of course in the unbroken phase, there are simply four diagonal conductivities for all the four bulk gauge fields. In the broken phase there are however interesting new phenomena. In particular there are now off-diagonal conductivities that do not simply vanish. In addition we have found that also the diagonal conductivities in the (1) - (2) sector, the one containing the type II Goldstone mode, have delta-function poles at zero frequency. In this sense this sector is still superconducting. Moreover, going to a decoupling basis for this sector leads to a very suggestive result: the conductivity develops a Drude-like peak characteristic of metals on top of the infinite DC conductivity. On the other hand Landau's criterion for superfluidity does not hold in this sector. Recall that this says that superfluidity takes place for flow velocities $v$ that are smaller than the critical velocity $v_{c}$ where $v_{c}=\min _{i} \omega_{i}(k) / k$ for all excitation branches $i$ and over all momenta $k$ [40, 41. For a type II Goldstone mode the critical flow velocity is clearly zero.

A second difference concerns the fate of the diffusive modes. In the unbroken phase there are simply four diffusive modes, one for each gauge field in the AdS bulk. In the broken phase there is one purely imaginary gapped 'pseudo-diffusive' mode in the $(0)-(3)$ sector, i.e. in the sector isomorphic to the $U(1)$ s-wave superfluid. Since there is still one unbroken $U(1)$ symmetry there is also a normal diffusive mode for the preserved $U(1)$ symmetry. In the $(1)-(2)$ sector we have however two diffusive modes in the unbroken phase. Going through the phase transition these two modes can pair up and move off the imaginary axis, becoming a pair of usual gapped quasinormal modes with real and imaginary parts in their frequencies. Generically the imaginary part of this gap is smaller (i.e. it lies closer to the real axis) then the gap of the purely imaginary mode in the $(0)-(3)$ sector. A large, generic perturbation will in its late time response pattern excite both the (0) - (3) and the (1) - (2) sector. The late time response of the $U(2)$ invariant order parameter $\sqrt{\left|\mathcal{O}_{1}\right|^{2}+\left|\mathcal{O}_{2}\right|^{2}}$ will therefore be dominated by these paired modes and show an oscillatory behavior in contrast to the response pattern of the order parameter in the $U(1)$ case [17].

Another remarkable QNM is the special gapped mode, i.e. the partner mode of the type II Goldstone boson. At very high temperatures this mode and the one which at $T=T_{c}$ leads to the sound mode are degenerate. As we lower the temperature the gap of these modes becomes different and, for $T<T_{c}$, it is expected that $\operatorname{Re}(\omega(k=0))$ for the Special Gapped mode is proportional to $q \mu[28,31]$. In particular we find $\omega \sim 1.1 \mu$ even if $q=1$ in our conventions. Unfortunately with the numerical methods employed in this paper we found it very difficult to study this mode and the discrepancy can therefore very well be a consequence of insufficient numerical accuracy. It is probably worth the effort to study this mode with alternative methods such as the relaxation method developed in [42, 43].

There are several generalizations of the $U(2)$ model that seem interesting and could be investigated in the future. A straightforward one would be to analyze the $p$-wave like instability commented in section 4 and to look for a stable background at low temperatures.

condensates was as well found in [39] 
The existence of a perturbation that becomes tachyonic means that the system will suffer a second order phase transition into a new phase, probably with the remaining $U(1)$ symmetry broken. Such an investigation is currently underway. A similar scenario has been found in [44, 45], in which a gauged version of the field theoretical sigma model undergoes a phase transition driven by an anisotropic vector condensate.

Another possible generalization would be to analyze the model when the backreaction onto the metric is taken into account. This introduces the energy-momentum tensor as an operator of the dual field theory and thus we expect the usual sound and shear modes to stem from bulk metric fluctuations. Moreover, this would allow us to obtain reliable results even at very low temperatures and for instance compute the density of superconducting charge densities at zero temperature, as well as $\mathcal{B}(T=0)$.

We have constructed here a simply model with type II Goldstone bosons using a "bottomup" strategy. It is however also interesting to ask if such models can be realized via "topdown" D-brane, string theory or M-theory constructions [46, 47, 48, 49, 50].

Another possible direction of research involves using the Fluid/Gravity correspondence [51] in order to derive the Hydrodynamic expansion of the current and upon including backreaction also the constitutive relation for the energy-momentum tensor. This will throw light on the hydrodynamic behavior of non-relativistic superfluids and in particular should result in the formulation of the hydrodynamics of relativistic type II Goldstone modes. Up to our knowledge this is not even known to the leading, i.e. zeroth order in derivatives.

Another direction of investigation concerns the Landau criterion of superfluidity. According to this criterion, the dispersion relation 105 prevents the system from accommodating a superflow. Therefore, even though $\sigma_{11}$ and $\sigma_{22}$ are superconducting, as soon as a nonvanishing supercurrent/superflow is switched on the system should in principle be taken out of the superfluid phase. It should be noted however that in a holographic superfluid the condensate and its flow are of leading order in a large $N$ expansion and the excitation spectrum, the QNMs, are subleading. Therefore it seems not clear if Landau's criterion can be applied straightforwardly. It is known however that for the $U(1)$ superfluid there exists a critical superflow or a critical supercurrent above which the condensate vanishes [52, 53, 54]. It would be very interesting to analyze if this is so (along the lines of [54]) and also to study how the whole mechanism takes place. Such an investigation is currently underway [55].

Finally it is also interesting to ask the question if holographic models featuring Goldstone modes with higher order dispersion relation $\omega=c k^{n}$ with $n>2$ can be constructed.

\section{A Matrix valued Kramers-Kronig relation}

The generically matrix-valued spectral function is defined as

$$
\rho_{i j}(x)=\left\langle\left[\mathcal{O}_{i}(x), \mathcal{O}_{j}(0)\right]\right\rangle,
$$

where $\mathcal{O}_{i}$ are Hermitian operators. Its behavior under Hermitian conjugation is

$$
\rho(x)^{\dagger}=\rho(-x)=-\rho(x)^{t} .
$$


Correspondingly, the Fourier transform $\tilde{\rho}(k)=\int d^{4} x e^{-i k x} \rho(x)$ also satisfies a set of identities

$$
\tilde{\rho}(k)^{\dagger}=\tilde{\rho}(k)=-\tilde{\rho}(-k)^{t} .
$$

In particular this means that the diagonal components are real and antisymmetric under $k \rightarrow-k$. One may also be interested in the behavior under $\omega \rightarrow-\omega$. We take now $k=(\omega, \mathbf{q})$. For theories with rotational invariance the spectral function can depend only on $\mathbf{q}^{2}$. Consequently the diagonal components will also be real and odd in $\omega$

$$
\rho_{i i}\left(\omega, \mathbf{q}^{2}\right)=\rho_{i i}\left(\omega, \mathbf{q}^{2}\right)^{*}=-\rho_{i i}\left(-\omega, \mathbf{q}^{2}\right) .
$$

For the off-diagonal components however, only if one also imposes time reversal or parity symmetry can one prove that the off-diagonal entries must be either even or odd functions of the frequency. In the present case time reversal symmetry is broken by the presence of the chemical potential. Further constraints can however by obtained by supposing that the theory is invariant under $\mathbf{x} \rightarrow-\mathbf{x}$. For an odd number of spatial dimensions we could use the parity operators $P$ to take $\mathbf{x} \rightarrow-\mathbf{x}$. In the two spatial dimensions we study in this paper we can take $P$ to by a rotation by $\pi$ (for an arbitrary even number of spatial dimensions $D=2 n$ we could take the angle $\pi$ for all the rotations in the $i, i+1$-th plane for all $i \leq n)$. This P-operator acts as $P \mathcal{O}_{i}(t, \mathbf{x}) P^{-1}=\sigma_{i} \mathcal{O}_{i}(t,-\mathbf{x})$ with $\sigma_{i}= \pm 1$. In odd spatial dimensions $\sigma_{i}$ is the parity of the operator. In even spatial dimension $\sigma_{i}=-1$ if $\mathcal{O}_{i}$ is the component of a spatial vector. Hence

$$
P\left[\rho_{i j}(t, \mathbf{x})\right]=\sigma_{i} \sigma_{j} \rho_{i j}(t,-\mathbf{x}) .
$$

P-invariance implies $\rho_{i j}(t, \mathbf{x})=\sigma_{i} \sigma_{j} \rho_{i j}(t,-\mathbf{x})$, which for the Fourier transform implies that

$$
\tilde{\rho}_{i j}(\omega, \mathbf{q})=-\sigma_{i} \sigma_{j} \tilde{\rho}_{i j}(-\omega, \mathbf{q})^{*} .
$$

So the off-diagonal entries are either odd or even functions of $\omega$ depending on the signs $\sigma_{i}$. In the case where the fields transform in the same way under the parity operator this means that the real (imaginary) part of the off-diagonal components is an odd (even) function of the frequency.

From the spectral function, as defined in (109) we can define two causal propagators, namely the retarded and advanced Green's functions

$$
\begin{aligned}
& G_{R}(x)=-i \Theta(t) \rho(x), \\
& G_{A}(x)=i \Theta(-t) \rho(x),
\end{aligned}
$$

where $x=(t, \mathbf{x})$. Using (111), one can prove the following relation among the Fourier transforms of these

$$
\tilde{G}_{R}(k)=\tilde{G}_{R}(-k)^{*}=\tilde{G}_{A}(k)^{\dagger} .
$$

From here, we see that the real (imaginary) part, $\operatorname{Re}\left(G_{R}\right)\left(\operatorname{Im}\left(G_{R}\right)\right)$, is even (odd) under $k \rightarrow-k$. We can compute the Fourier transform of the retarded Green's function, which is 
given by the convolution of the Fourier transform of the Heaviside step function $\tilde{\Theta}(\omega)$ with the Fourier transform of the spectral function $\tilde{\rho}(k)$,

$$
\tilde{G}_{R}(\omega, \mathbf{q})=-i \int_{-\infty}^{\infty} \tilde{\Theta}(\omega-\mu) \tilde{\rho}(\mu, \mathbf{q}) \frac{d \mu}{2 \pi} .
$$

Using the Fourier transform of the step function

$$
\tilde{\Theta}(\omega)=\frac{i}{\omega+i \epsilon},
$$

and the Sokhatsky-Weierstrass theorem we get

$$
\tilde{G}_{R}(\omega, \mathbf{q})=\mathcal{P} \int_{-\infty}^{\infty} \frac{\tilde{\rho}\left(\omega^{\prime}, \mathbf{q}\right)}{\omega-\omega^{\prime}} \frac{d \omega^{\prime}}{2 \pi}-\frac{i}{2} \tilde{\rho}(\omega, \mathbf{q})
$$

where $\mathcal{P}$ denotes the principle value. From the Hermiticity of $\tilde{\rho}(k)$ we see that we can regard (119) as a split of $\tilde{G}^{R}(k)$ into its Hermitian and anti-Hermitian parts, and find that the spectral function can be computed from the anti-Hermitian part of the Fourier transform of the retarded Green's function

$$
\tilde{\rho}(k)=i\left[\tilde{G}_{R}(k)-\tilde{G}_{R}(k)^{\dagger}\right] \equiv 2 i \tilde{G}_{R}^{(A)}(k),
$$

where the $(A)$ stands for anti-Hermitian ${ }^{10}$. Plugging this back into (119) and taking the Hermitian part $(H)$ on both sides we arrive at

$$
\tilde{G}_{R}^{(H)}(\omega)=\frac{i}{\pi} \mathcal{P} \int_{-\infty}^{\infty} \frac{G_{R}^{(A)}\left(\omega^{\prime}\right)}{\omega-\omega^{\prime}} d \omega^{\prime},
$$

which is nothing but the Kramers-Krönig relation for the matrix Green's function. It is complemented by the conjugate relation interchanging the Hermitian and anti-Hermitian parts. Imposing P-invariance and using (114) and (119) if follows that the Green's function satisfies

$$
\tilde{G}_{i j}^{R}(\omega, \mathbf{q})=\sigma_{i} \sigma_{j} \tilde{G}_{i j}^{R}(-\omega, \mathbf{q})^{*} .
$$

This constrains the QNM spectrum. Taking for example a diagonal Green's function with $i=j$ and writing it as a sum over quasinormal frequencies [33, 56] one seems that the quasinormal frequencies have to come either in pairs obeying $\omega_{n}$ and $\tilde{\omega}_{n}=-\omega_{n}^{*}$ or are confined to lie on the imaginary axis. The residues of the pairs are related by complex conjugation and the purely imaginary ones have to have also purely imaginary residue.

\section{B Solving the fluctuation equations}

The (1) - (2) sector of the gauged model in the broken phase consists of a system of coupled equations 83 - 90 . In order to extract the spectrum of quasinormal modes we

\footnotetext{
${ }^{10}$ Using 117 we can always work with retarded Green's functions $G_{R}$.
} 
made use of the techniques detailed in [15, 34, where a method to compute the poles of the Green functions in terms of non-gauge invariant fields was developed. The quasinormal frequencies are given by the zeroes of the determinant of the field matrix spanned by a maximal set of linearly independent solutions satisfying infalling boundary conditions on the horizon evaluated at the boundary.

Imposing infalling boundary conditions, the near horizon behavior of the fields solving the mentioned equations reads

$$
\begin{aligned}
\alpha & =(\rho-1)^{\kappa}\left(\alpha_{(0)}+\alpha_{(1)}(\rho-1)+\ldots\right), \\
\beta & =(\rho-1)^{\kappa}\left(\beta_{(0)}+\beta_{(1)}(\rho-1)+\ldots\right), \\
a_{t}^{(i)} & =(\rho-1)^{\kappa+1}\left(a_{t(0)}^{(i)}+a_{t(1)}^{(i)}(\rho-1)+\ldots\right), \\
a_{x}^{(i)} & =(\rho-1)^{\kappa}\left(a_{x(0)}^{(i)}+a_{x(1)}^{(i)}(\rho-1)+\ldots\right),
\end{aligned}
$$

where $\kappa=-i \omega / 3$ and $i=1,2$. Since the system is subject to two constraints, we can only choose four of the six parameters at the horizon. Without loss of generality, solutions can be parametrized by $\left\{\alpha_{(0)}, \beta_{(0)}, a_{x(0)}^{(i)}\right\}$. In this way it is possible to construct four independent solutions to the field equations. We can label them as $I, I I, I I I, I V$.

Two additional solutions, $V, V I$, can be obtained by performing gauge transformations of the trivial solution,

$$
\begin{aligned}
& \alpha \rightarrow 0, \beta \rightarrow i \frac{\lambda_{1} \Psi}{2}, a_{x}^{(1)} \rightarrow-k \lambda_{1}, a_{x}^{(2)} \rightarrow 0, a_{t}^{(1)} \rightarrow \omega \lambda_{1}, a_{t}^{(2)} \rightarrow i \Theta \lambda_{1}, \\
& \alpha \rightarrow i \frac{\lambda_{2} \Psi}{2}, \beta \rightarrow 0, a_{x}^{(1)} \rightarrow 0, a_{x}^{(2)} \rightarrow-k \lambda_{2}, a_{t}^{(1)} \rightarrow-i \Theta \lambda_{2}, a_{t}^{(2)} \rightarrow \omega \lambda_{2},
\end{aligned}
$$

where $\lambda_{i}$ are arbitrary constants. Notice that these pure gauge solutions are not algebraic since they have a nontrivial dependence on the bulk coordinate $\rho$.

The most general solution for each field $\varphi_{i}=\left\{\tilde{\alpha}, \tilde{\beta}, a_{t}^{(i)}, a_{x}^{(i)}\right\}$ is given by a linear combination of the above solutions, including the pure gauge modes,

$$
\varphi_{i}=c_{I} \varphi_{i}^{I}+c_{I I} \varphi_{i}^{I I}+c_{I I I} \varphi_{i}^{I I I}+c_{I V} \varphi_{i}^{I V}+c_{V} \varphi_{i}^{V}+c_{V I} \varphi_{i}^{V I}
$$

where we have defined $\{\tilde{\alpha}(\rho), \tilde{\beta}(\rho)\}=\{\rho \alpha(\rho), \rho \beta(\rho)\}$. This convenient choice allows us to identify the asymptotic boundary values $\varphi_{i}$ with the sources of the gauge invariant operators of the dual field theory.

As shown in [15], the poles of the retarded Green functions will be given by the values of the frequency for which the determinant of the matrix spanned by $\varphi_{i}^{N}$ vanishes asymptoti- 
cally. Expanding the determinant and evaluating it at a cutoff $\rho=\Lambda$, it reads

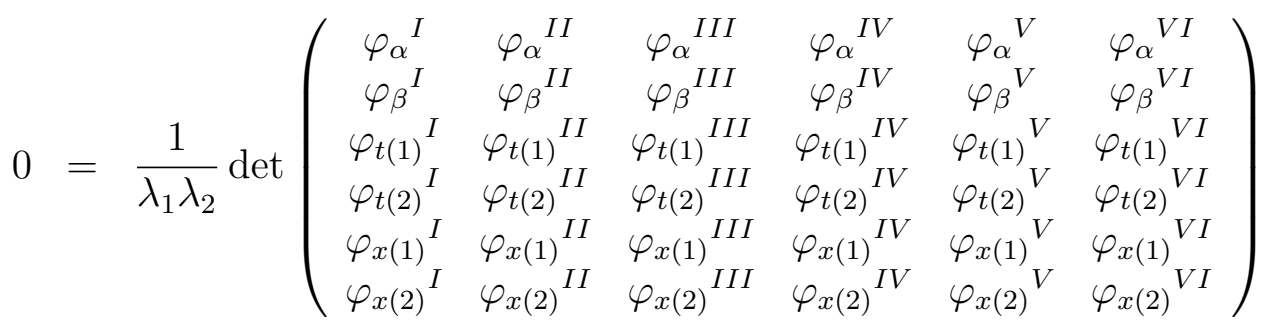

$$
\begin{aligned}
& =\omega^{2} \operatorname{det}\left(\begin{array}{cccc}
\varphi_{\alpha}^{I} & \varphi_{\alpha}^{I I} & \varphi_{\alpha}^{I I I} & \varphi_{\alpha}^{I V} \\
\varphi_{\beta}^{I} & \varphi_{\beta}^{I I} & \varphi_{\beta}^{I I} & \varphi_{\beta}^{I V} \\
\varphi_{x(1)}^{I} & \varphi_{x(1)}^{I I} & \varphi_{x(1)}^{I I I} & \varphi_{x(1)}^{I V} \\
\varphi_{x(2)}^{I} & \varphi_{x(2)}^{I I} & \varphi_{x(2)}^{I I I} & \varphi_{x(2)}^{I V}
\end{array}\right)+\omega k \operatorname{det}\left(\begin{array}{cccc}
\varphi_{\alpha}^{I} & \varphi_{\alpha}^{I I} & \varphi_{\alpha}^{I I I} & \varphi_{\alpha}^{I V} \\
\varphi_{\beta}^{I} & \varphi_{\beta}^{I I} & \varphi_{\beta}^{I I I} & \varphi_{\beta}^{I V} \\
\varphi_{t(1)}^{I} & \varphi_{t(1)}^{I I} & \varphi_{t(1)}^{I I I} & \varphi_{t(1)}^{I V} \\
\varphi_{x(2)}^{I} & \varphi_{x(2)}^{I I} & \varphi_{x(2)}^{I I I} & \varphi_{x(2)}^{I V}
\end{array}\right) \\
& -\omega k \operatorname{det}\left(\begin{array}{cccc}
\varphi_{\alpha}^{I} & \varphi_{\alpha}^{I I} & \varphi_{\alpha}^{I I I} & \varphi_{\alpha}^{I V} \\
\varphi_{\beta}^{I} & \varphi_{\beta}^{I I} & \varphi_{\beta}^{I I I} & \varphi_{\beta}^{I V} \\
\varphi_{t(2)}^{I V} & \varphi_{t(2)}^{I I} & \varphi_{t(2)}^{I I I} & \varphi_{t(2)}^{I V} \\
\varphi_{x(1)}^{I} & \varphi_{x(1)}^{I I} & \varphi_{x(1)}^{I I I} & \varphi_{x(1)}^{I V}
\end{array}\right)+k^{2} \operatorname{det}\left(\begin{array}{cccc}
\varphi_{\alpha}^{I} & \varphi_{\alpha}^{I I} & \varphi_{\alpha}^{I I I} & \varphi_{\alpha}^{I V} \\
\varphi_{\beta}^{I} & \varphi_{\beta}^{I I} & \varphi_{\beta}^{I I I} & \varphi_{\beta}^{I V} \\
\varphi_{t(1)}^{I V} & \varphi_{t(1)}^{I I} & \varphi_{t(1)}^{I I I} & \varphi_{t(1)}^{I V} \\
\varphi_{t(2)}^{I} & \varphi_{t(2)}^{I I} & \varphi_{t(2)}^{I I I} & \varphi_{t(2)}^{I V}
\end{array}\right),
\end{aligned}
$$

where the background boundary conditions $\Theta(\Lambda)=0$ and $\Lambda \Psi=0$ have been already imposed. This absence of background sources for the corresponding operators makes the point $(\omega, k)=(0,0)$ a trivial solution to the vanishing determinant condition, which ensures the existence of a hydrodynamic mode. Notice also that the point $(\omega, k)=(0,0)$ is a double solution to the previous determinant equation.

Solutions to the equations of motion and to the determinant condition (130) have been computed numerically. It has been checked that the election of solution basis, i.e. of initial values of the free parameters, does not affect the result.

\section{Acknowledgments}

We would like to thank T. Brauner, J. Gauntlett, A. G. Grushin, I. Shovkovy, A. Schmitt and M.A. H. Vozmediano, T. Wiseman for enjoyable and useful discussions. I. A. is supported by the Israel Science Foundation under grants no. 392/09 and 495/11. A. J., L. M. and K. L. are supported by Plan Nacional de Altas Energías FPA 2009-07890, Consolider Ingenio 2010 CPAN CSD200-00042 and HEP-HACOS S2009/ESP-2473. L.M. has been supported by FPI-fellowship BES-2010-041571. A. J. has been supported by FPU fellowship AP20105686. D. A. thanks the FRont Of pro-Galician Scientists for unconditional support. D. A. and I. S. would like to thank the HIPM for reminding them how fun working in physics can be.

\section{References}

[1] S. S. Gubser, "Breaking an Abelian gauge symmetry near a black hole horizon," Phys. Rev. D 78 (2008) 065034 arXiv:0801.2977 [hep-th]]. 
[2] S. A. Hartnoll, C. P. Herzog and G. T. Horowitz, "Building a Holographic Superconductor," Phys. Rev. Lett. 101 (2008) 031601 arXiv:0803.3295 [hep-th]].

[3] S. A. Hartnoll, C. P. Herzog and G. T. Horowitz, "Holographic Superconductors," JHEP 0812 (2008) 015 arXiv:0810.1563 [hep-th]].

[4] S. S. Gubser and S. S. Pufu, "The Gravity dual of a p-wave superconductor," JHEP 0811 (2008) 033 [arXiv:0805.2960 [hep-th]].

[5] F. Benini, C. P. Herzog, R. Rahman and A. Yarom, "Gauge gravity duality for d-wave superconductors: prospects and challenges," JHEP 1011 (2010) 137 arXiv:1007.1981 [hep-th]].

[6] J. -W. Chen, Y. -J. Kao, D. Maity, W. -Y. Wen and C. -P. Yeh, "Towards A Holographic Model of D-Wave Superconductors," Phys. Rev. D 81 (2010) 106008 arXiv:1003.2991 [hep-th]].

[7] G. T. Horowitz, "Introduction to Holographic Superconductors," arXiv:1002.1722 [hepth].

[8] M. Kaminski, "Flavor Superconductivity \& Superfluidity," Lect. Notes Phys. 828 (2011) 349 arXiv:1002.4886 [hep-th]].

[9] D. T. Son and A. O. Starinets, "Minkowski space correlators in AdS / CFT correspondence: Recipe and applications," JHEP 0209 (2002) 042 [hep-th/0205051].

[10] C. P. Herzog and D. T. Son, "Schwinger-Keldysh propagators from AdS/CFT correspondence," JHEP 0303 (2003) 046 [hep-th/0212072].

[11] G. T. Horowitz and V. E. Hubeny, "Quasinormal modes of AdS black holes and the approach to thermal equilibrium," Phys. Rev. D 62 (2000) 024027 hep-th/9909056].

[12] D. Birmingham, I. Sachs and S. N. Solodukhin, "Conformal field theory interpretation of black hole quasinormal modes," Phys. Rev. Lett. 88 (2002) 151301 hep-th/0112055].

[13] E. Berti, V. Cardoso and A. O. Starinets, "Quasinormal modes of black holes and black branes," Class. Quant. Grav. 26 (2009) 163001 arXiv:0905.2975 [gr-qc]].

[14] K. Landsteiner, "The Sound of Strongly Coupled Field Theories: Quasinormal Modes In AdS," AIP Conf. Proc. 1458 (2011) 174 [arXiv:1202.3550 [gr-qc]].

[15] I. Amado, M. Kaminski and K. Landsteiner, "Hydrodynamics of Holographic Superconductors," JHEP 0905 (2009) 021 arXiv:0903.2209 [hep-th]].

[16] A. Yarom, "Fourth sound of holographic superfluids," JHEP 0907 (2009) 070 arXiv:0903.1353 [hep-th]]. 
[17] M. J. Bhaseen, J. P. Gauntlett, B. D. Simons, J. Sonner and T. Wiseman, "Holographic Superfluids and the Dynamics of Symmetry Breaking," arXiv:1207.4194 [hep-th].

[18] B.I. Halperin, "Dynamic properties of the multicomponent Bose fluid," Phys. Rev. B 11, 178190 (1975).

[19] T. Schafer, D. T. Son, M. A. Stephanov, D. Toublan and J. J. M. Verbaarschot, "Kaon condensation and Goldstone's theorem," Phys. Lett. B 522 (2001) 67 [hep-ph/0108210].

[20] V. A. Miransky and I. A. Shovkovy, "Spontaneous symmetry breaking with abnormal number of Nambu-Goldstone bosons and kaon condensate," Phys. Rev. Lett. 88 (2002) 111601 hep-ph/0108178].

[21] V. G. Filev, C. V. Johnson and J. P. Shock, "Universal Holographic Chiral Dynamics in an External Magnetic Field," JHEP 0908 (2009) 013 [arXiv:0903.5345 [hep-th]].

[22] T. Brauner, "Spontaneous Symmetry Breaking and Nambu-Goldstone Bosons in Quantum Many-Body Systems," Symmetry 2 (2010) 609 arXiv:1001.5212 [hep-th]].

[23] H. B. Nielsen and S. Chadha, "On How to Count Goldstone Bosons," Nucl. Phys. B 105 (1976) 445.

[24] H. Watanabe and T. Brauner, "On the number of Nambu-Goldstone bosons and its relation to charge densities," Phys. Rev. D 84 (2011) 125013 arXiv:1109.6327 [hep$\mathrm{ph}]]$.

[25] H. Watanabe and H. Murayama, "Unified Description of Nambu-Goldstone Bosons without Lorentz Invariance," Phys. Rev. Lett. 108 (2012) 251602 arXiv:1203.0609 [hepth]].

[26] H. Watanabe and H. Murayama, "Redundancies in Nambu-Goldstone Bosons," arXiv:1302.4800 [cond-mat.other].

[27] Y. Hidaka, "Counting rule for Nambu-Goldstone modes in nonrelativistic systems," Phys. Rev. Lett. 110 (2013) 091601 arXiv:1203.1494 [hep-th].

[28] A. Kapustin, "Remarks on nonrelativistic Goldstone bosons," arXiv:1207.0457 [hep-ph].

[29] Z. Q. Li, E. A. Henriksen, Z. Jiang, Z. Hao, M. C. Martin, P. Kim, H. L. Stormer and D. N. Basov, "Dirac charge dynamics in graphene by infrared spectroscopy," Nature Physics 4, 532 - 535 (2008).

[30] T. Brauner, "Spontaneous symmetry breaking in the linear sigma model at finite chemical potential: One-loop corrections," Phys. Rev. D 74 (2006) 085010 [hep-ph/0607102].

[31] A. Nicolis and F. Piazza, "A relativistic non-relativistic Goldstone theorem: gapped Goldstones at finite charge density," Phys. Rev. Lett. 110 (2013) 011602 [arXiv:1204.1570 [hep-th]]. 
[32] I. R. Klebanov and E. Witten, "AdS / CFT correspondence and symmetry breaking," Nucl. Phys. B 556 (1999) 89 [hep-th/9905104]. in which $\psi_{1}$ is

[33] I. Amado, C. Hoyos-Badajoz, K. Landsteiner and S. Montero, "Hydrodynamics and beyond in the strongly coupled N=4 plasma," JHEP 0807 (2008) 133 arXiv:0805.2570 [hep-th]].

[34] M. Kaminski, K. Landsteiner, J. Mas, J. P. Shock and J. Tarrio, "Holographic Operator Mixing and Quasinormal Modes on the Brane," JHEP 1002 (2010) 021 arXiv:0911.3610 [hep-th]].

[35] R. A. Davison and A. O. Starinets, "Holographic zero sound at finite temperature," Phys. Rev. D 85 (2012) 026004 [arXiv:1109.6343 [hep-th]].

[36] F. Bigazzi, A. L. Cotrone, D. Musso, N. P. Fokeeva and D. Seminara, "Unbalanced Holographic Superconductors and Spintronics," JHEP 1202 (2012) 078 arXiv:1111.6601 [hep-th]].

[37] S. A. Hartnoll, "Lectures on holographic methods for condensed matter physics," Class. Quant. Grav. 26 (2009) 224002 [arXiv:0903.3246 [hep-th]].

[38] S. C. 'Zhang. 1997. " A Unified Theory Based on SO(5) Symmetry of Superconductivity and Antiferromagnetism," Science,275,1089

[39] S. Uchino, M. Kobayashi and M. Ueda, "Bogoliubov Theory and Lee-Huang-Yang Corrections in Spin-1 and Spin-2 Bose-Einstein Condensates in the Presence of the Quadratic Zeeman Effect," Phys. Rev. A 81 (2010) 063632.

[40] I. M. Khalatnikov, "An Introduction to the Theory of Superfluidity," Advanced Book Classics, Westview Press.

[41] L. .D. Landau and E. M. Lifshitz, "Course of Theoretical Physcis, "Vol. 9, Statistical Physics, Part 2, Chap. III, Pergamon Press.

[42] C. Hoyos-Badajoz, K. Landsteiner and S. Montero, "Holographic meson melting," JHEP 0704 (2007) 031 [hep-th/0612169].

[43] M. Kaminski, K. Landsteiner, F. Pena-Benitez, J. Erdmenger, C. Greubel and P. Kerner, "Quasinormal modes of massive charged flavor branes," JHEP 1003 (2010) 117 arXiv:0911.3544 [hep-th]].

[44] V. P. Gusynin, V. A. Miransky and I. A. Shovkovy, "Spontaneous rotational symmetry breaking and roton - like excitations in gauged sigma model at finite density," Phys. Lett. B 581, 82 (2004) hep-ph/0311025].

[45] V. P. Gusynin, V. A. Miransky and I. A. Shovkovy, "Surprises in nonperturbative dynamics in sigma-model at finite density," Mod. Phys. Lett. A 19 (2004) 1341 hepph/0406219]. 
[46] M. Ammon, J. Erdmenger, M. Kaminski and P. Kerner, "Superconductivity from gauge/gravity duality with flavor," Phys. Lett. B 680 (2009) 516 arXiv:0810.2316 [hepth]].

[47] J. P. Gauntlett, J. Sonner and T. Wiseman, "Holographic superconductivity in MTheory," Phys. Rev. Lett. 103 (2009) 151601 [arXiv:0907.3796 [hep-th]].

[48] S. S. Gubser, C. P. Herzog, S. S. Pufu and T. Tesileanu, "Superconductors from Superstrings," Phys. Rev. Lett. 103 (2009) 141601 [arXiv:0907.3510 [hep-th]].

[49] N. Bobev, N. Halmagyi, K. Pilch and N. P. Warner, "Supergravity Instabilities of Non-Supersymmetric Quantum Critical Points," Class. Quant. Grav. 27 (2010) 235013 arXiv:1006.2546 [hep-th]].

[50] N. Bobev, A. Kundu, K. Pilch and N. P. Warner, "Minimal Holographic Superconductors from Maximal Supergravity," JHEP 1203 (2012) 064 [arXiv:1110.3454 [hep-th]].

[51] S. Bhattacharyya, V. EHubeny, S. Minwalla and M. Rangamani, "Nonlinear Fluid Dynamics from Gravity," JHEP 0802 (2008) 045 [arXiv:0712.2456 [hep-th]].

[52] C. P. Herzog, P. K. Kovtun and D. T. Son, "Holographic model of superfluidity," Phys. Rev. D 79 (2009) 066002 arXiv:0809.4870 [hep-th]].

[53] P. Basu, A. Mukherjee and H. -H. Shieh, "Supercurrent: Vector Hair for an AdS Black Hole," Phys. Rev. D 79 (2009) 045010 arXiv:0809.4494 [hep-th]].

[54] D. Arean, M. Bertolini, J. Evslin and T. Prochazka, "On Holographic Superconductors with DC Current," JHEP 1007 (2010) 060 [arXiv:1003.5661 [hep-th]].

[55] In preparation.

[56] M. A. Stephanov and Y. Yin, "Conductivity and quasinormal modes in holographic theories," JHEP 1202 (2012) 017 [arXiv:1111.5303 [hep-ph]]. 